\title{
Radar Measurements of Snow: Experiment and Analysis
}

John R. Kendra, Member, IEEE, Kamal Sarabandi, Senior Member, IEEE, and Fawwaz T. Ulaby, Fellow, IEEE

\begin{abstract}
This paper considers two specific types of experiments conducted to improve our understanding of radar backscatter from snow-covered ground surfaces. The first experiment involves radar backscatter measurements at $\mathrm{C}$ - and $\mathrm{X}$-band of artificial snow of varying depths. The relatively simple target characteristics, combined with an exhaustive ground truth effort, make the results of this experiment especially amenable to comparison with predictions based on theoretical methods for modeling volume-scattering media. It is shown that both conventional and dense-medium radiative transfer models fail to adequately explain the observed results. A direct polarimetric inversion approach is described by which the characteristics of the snow medium are extracted from the measured data. The second type of experiment examined in this study involves diurnal backscatter measurements that were made contemporaneously with detailed measurements of the snow-wetness depth profiles of the observed scene. These data are used to evaluate the capability of a recently proposed algorithm for snow wetness retrieval from polarimetric synthetic aperture radar (SAR) measurements, which has hithertofore been applied only to data from very complex and extended mountainous terrains.
\end{abstract}

\section{INTRODUCTION}

A GREAT deal of experimental and theoretical work has been done pertaining to the radar response of snow or, by extension, dense random media in the theoretical realm, for which snow is perhaps the most representative natural example. The aim of this work, and what is true of any remotesensing research, is to develop the capability to characterize in some way, to a greater or lesser degree, the remote target from the sensor response(s) alone. We may identify three levels of such characterization, which we list in order of increasing power of characterization but decreasing level of reliability.

- Level 1: Empirical models are used to infer or predict information about the target characteristics. Recent examples include radar and radiometer algorithms for discriminating wet snow-covered terrain from other types of terrain [1], a hybrid empirical/theoretical approach for estimating radar clutter at millimeter wave due to certain types of terrain [2], and an approach for classifying snow cover states (dry/wet/refrozen) [3].

- Level 2: Classification is performed based on the apparent scattering mechanisms inherent in the target, as identified

Manuscript received October 3, 1995; revised April 7, 1997. This work was supported in part by ONR under Contract N00014-95-1-0736.

J. R. Kendra is with Raytheon Systems Company, Intelligence and Aircraft Integration Systems, Dallas, TX 75266-0023 USA.

K. Sarabandi and F. T. Ulaby are with the Radiation Laboratory, Department of Electrical Engineering and Computer Science, The University of Michigan, Ann Arbor, MI 48109-2122 USA (e-mail: saraband@eecs.umich.edu).

Publisher Item Identifier S 0196-2892(98)02851-4. through analysis of the character of the target Mueller matrix [4], [5]. Such techniques have reportedly been successful in demarcating areas consisting of primarily urban targets, slightly rough targets like oceans and lava flows, and parks and vegetated areas. It has also been shown that such a method may allow discrimination between relatively younger and older lava flows [6]. These techniques have also lately been suggested as a means for determining wetness levels in snow [7], [8].

- Level 3: At this level, tools from the previous two may be used, but the central characteristic is the use of a theoretical model that is assumed to generate reasonably high fidelity predictions of sensor responses for a given set of physical parameters that is assumed to constitute an accurate description of the target. An example of this type of approach is a neural-net-driven inversion algorithm intended to allow retrieval of snow parameter from radar and radiometer sensor responses which is trained using a dense, medium radiative transfer (DMRT) model [9].

It is obvious from the descriptions that, where greater understanding of the electromagnetic interaction with a material is present, the potential for information retrieval through remotely sensed data is greater. The critical issue becomes then testing the validity of theoretical models through careful experiments.

In the present study, an attempt is made to address this issue, with respect to the radar response of snow. There exists already numerous experimental studies of snow in the literature, both at microwave frequencies [10]-[14], and at millimeter-wave frequencies [15], [16]. Using such experimental data for the purpose of evaluation of models is very difficult because of the need to carefully characterize the target. Even with precise characterization, substantial obstacles remain. A complex target can be described down to the finest details, but this still leaves the problem of correctly modeling the behavior of all of these features, electromagnetically speaking, and all of their interactions with each other.

Consider a typical target of snow-covered ground. Parameters that must be potentially considered include the following.

- Parameters associated with the top surface of the snow (rough surface).

- The snow itself: density and particle size distribution; vertical distributions of these properties within the snowpack.

- Snow wetness, when present, may be a very complex function of time and depth. Examples of this are presented in later sections of this paper. 
- The ground beneath: its dielectric constant, roughness parameters, and local slope.

For experiments at millimeter-wave frequencies, the number of parameters may be somewhat reduced. The careful experiments in [17] demonstrate that the rate of attenuation is relatively large, and therefore, properties of the underlying ground are unimportant, except when the snow layer is dry and the snow depth only a few centimeters [18]. Thus, at millimeter-wave frequencies, the snow may often be approximated as a half-space. The price for this, however, is that sensitivity to snow microstructure and other smaller scale features is heightened, for which accurate characterization may be very difficult.

Microwave frequencies offer the most potential for the retrieval of gross snow properties, such as depth or water equivalent, parameters that are especially important for hydrological applications. For microwave frequencies, generally, most of the parameters mentioned above need to be considered. Thus, it is in practice difficult to address theoretical predictions in an unambiguous fashion with field experiments.

The present study describes radar backscatter experiments on snow at $\mathrm{C}$ - and $\mathrm{X}$-band. In these experiments, the use of artificially produced snow allowed an unusually high degree of confidence in the exact character of the target snowpack. Two different kinds of experiments were conducted: experiments of the angular response of dry snow at various depths and experiments at a single angle over a partial diurnal cycle, during which complete profiles of water content as a function of depth and time were recorded using the Snow Probe [19]. A complete description of the experiment is presented in Section II. In Section III, the results of the backscatter experiments are presented and analyzed, with respect to certain models. In Section IV, the results of this diurnal experiment and another previous diurnal experiment are presented and discussed, with respect to a proposed inversion algorithm for snow wetness.

\section{EXPERIMENT DESCRIPTION}

This section describes scatterometer experiments that were performed at $\mathrm{C}$ - and $\mathrm{X}$-band (5.3 and $9.5 \mathrm{GHz}$, respectively) on snowpacks comprised artificial snow. The experimental site was the Mt. Brighton Ski Area, Brighton, MI, during February and March of 1993. The radars used were truck-mounted, fully polarimetric, network-analyzer-based systems using horn antennas. Detailed descriptions of the systems are available elsewhere [20], [21].

The target was an area of ground covering approximately $18 \times 30 \mathrm{~m}$. On the average, 60 independent spatial measurements were taken with each angular measurement to reduce the variance due to fading in the estimate of the mean backscatter. Additional frequency averaging was available from the bandwidths used in the channels, 400 and $500 \mathrm{MHz}$ in the $\mathrm{C}$ - and $\mathrm{X}$-band channels, respectively. Calibration was performed using a 14-in sphere and a differential Mueller matrix algorithm suitable for measurements of distributed targets [22].

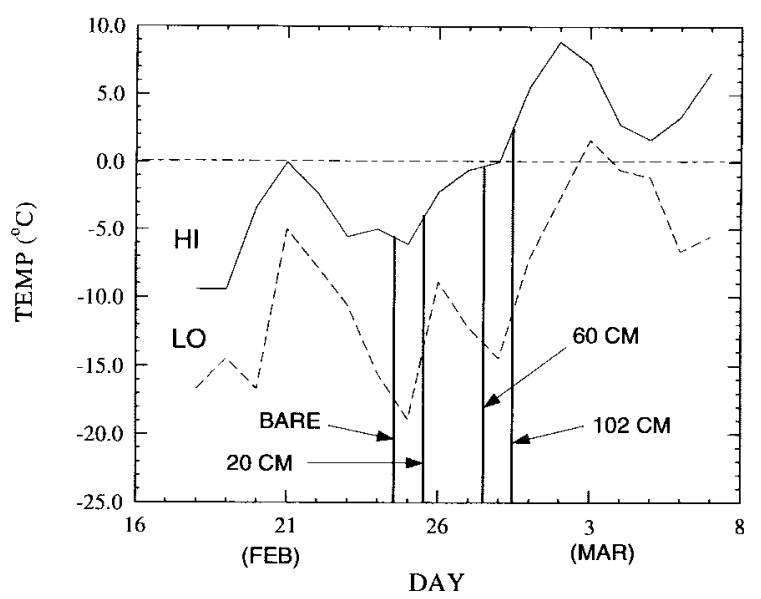

Fig. 1. Temperature variations during the course of the radar experiments on artificial snow. "Lo" (dashed curve) indicates daily low temperatures; "HI" (solid curve) indicates daily high temperatures.

Two types of experiments were performed. The first consisted of backscatter measurements at incidence angles of $20-60^{\circ}$ on bare ground, which were then repeated for three progressively deeper layers of artificial snow over the ground. This sequence was performed over a six-day period from February 24 to March 1. A major goal of the experiment was to simplify, insofar as was possible, the nature of the target to allow as unambiguous a comparison with theory as possible. One goal we had was insuring that there would be no wetness present in the target snowpacks, so that we would not have to deal with this complicating factor in our interpretation. We therefore conducted all of the experiments during the night. As may be seen from the daily temperatures plotted in Fig. 1, the temperature during the data-takes averaged $-15^{\circ} \mathrm{C}$ and was always below $-10^{\circ} \mathrm{C}$. Snow probe measurements verified that there was, to the accuracy of the measurement, essentially no liquid water present in the snowpack. An additional fortuitous circumstance, which may also be observed in Fig. 1, was that the temperature was very low in the week preceding the experiment, leading to a generally frozen environment that was unrelieved during the entire experiment, as even the temperature highs did not go above the freezing mark until the day following the final experiment.

The bare ground target was prepared by using earth-moving equipment to remove all of the existing snow, natural and artificial, and then to scrape away any grass or other vegetation on the target plot. The dielectric constant of the ground was measured using a microwave field-portable dielectric probe [23] operating at C-band. The average dielectric was found, from 25 separate measurements to be $4.6 \pm 1.1$ (the imaginary part is not estimated accurately through this technique). The bare ground roughness was measured using a laser profilometer. Seven $95-\mathrm{cm}$ linear transects were measured; the step size was $3 \mathrm{~mm}$. The average rms height of the surface was found to be $s=0.32 \pm 0.08 \mathrm{~cm}$, and the correlation length was found to be $l=2.09 \pm 1.6 \mathrm{~cm}$.

The artificial snow was added in three progressively deeper layers. No chemical additives were used in the production of the snow, as is commonly done to increase production, which 
TABLE I

Dry SNOW Physical Properties

\begin{tabular}{l|l|l|l}
\hline \multicolumn{1}{c|}{ Ground } & \multicolumn{1}{c|}{ Snow Volume } & \multicolumn{1}{c}{ Snow Surface } & \multicolumn{1}{c}{ Snow Depths } \\
\hline$s=0.32 \mathrm{~cm}$ & $\bar{d}=0.27 \pm 0.11 \mathrm{~mm}$ & $s=0.45 \mathrm{~cm}$ & $d_{1}=20 \pm 6 \mathrm{~cm}$ \\
$l=2.09$ & $\bar{\varepsilon}=1.9-j 0.015$ & $\varepsilon_{r}^{\prime}=1.97$ & $d_{2}=60 \pm 10 \mathrm{~cm}$ \\
$\varepsilon_{r}^{\prime}=4.7$ (C-band) & $\rho_{s}=0.48 \mathrm{~g} / \mathrm{cm}^{3}$ & Period: $3.2 \mathrm{~cm}$ & $d_{3}=102 \pm 9 \mathrm{~cm}$ \\
& & Amplitude (p-p): $1.27 \mathrm{~cm}$ & \\
\hline
\end{tabular}
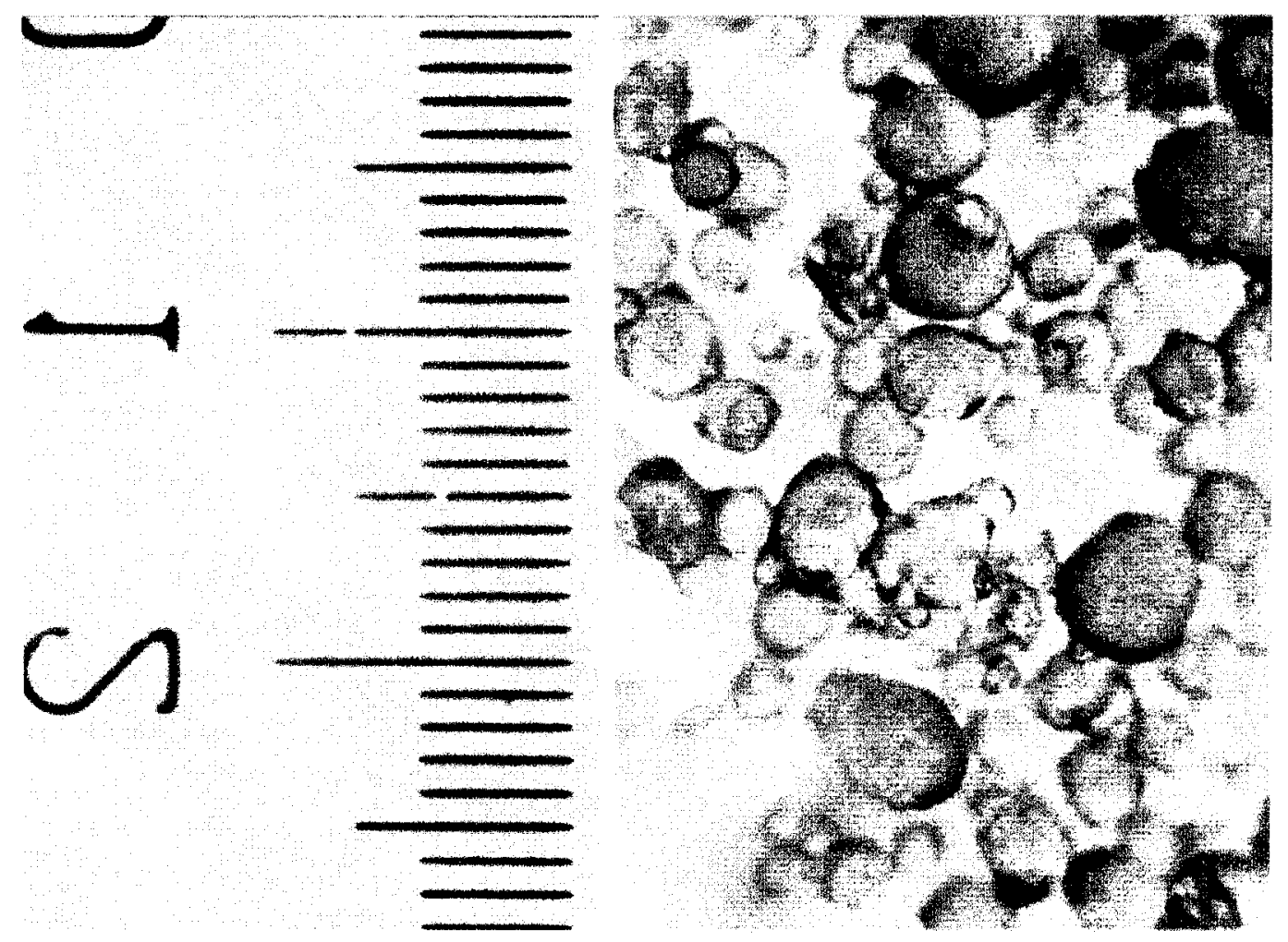

Fig. 2. Photograph of artificial snow particles. Major divisions of ruler shown are millimeters.

might have caused the dielectric constant of the particles to depart from that of water ice. The snow, once produced, was spread into a uniform layer using a grooming machine (piston bully) with extra wide treads to reduce the pressure exerted by the weight of the machine on the snowpack. A consequence of this process was that a characteristic groove pattern was imparted to the top of the snow, having a period of $3.2 \mathrm{~cm}$ and a peak-to-peak amplitude of $1.3 \mathrm{~cm}$, corresponding to an $\mathrm{rms}$ height of $0.449 \mathrm{~cm}$. The correlation length of such a surface is dependent on the direction with which it is computed relative to the grooves.

The three different depths of snow deposited were 20,60, and $102 \mathrm{~cm}$, with the standard deviations shown in Table I. The nature of artificial snow is that it consists of very small particles and has a very high density. The average density of the snow was $0.48 \mathrm{~g} / \mathrm{cm}^{3}$, and the average particle diameter was found to be $0.27 \mathrm{~mm}$. This combination of particle size and density are at the extreme ends of what would be found in nature, but not to the point of being unreasonable. We note, for example, that the density is very comparable to that reported for Alpine snowpacks in [7]; neither is the particle size outside of reported ranges [17], [24]. All of the target physical properties for the dry snow backscatter experiments are summarized in Table I.

It deserves to be emphasized that there were a number of elements present in this experimental effort that make it unusually well—and simply — characterized. The snow machines produced very uniform "snow" over the course of the experiment, and this was recorded and photographed. From Fig. 2, it is evident that the snowpack was in fact composed of discrete particles having a high degree of sphericity. Furthermore, there was little or no variation from this snow composition as a function of vertical position within the snowpack. This last point is owing not only to the uniform way in which the particles were produced, but also to the unbroken cold conditions that persisted throughout this experimental phase and therefore prevented any metamorphic activity associated with melting and refreezing within the earliest deposited snow. Finally, the action of the grooming machine on the top surface of the snow guaranteed that virtually identical roughness was 


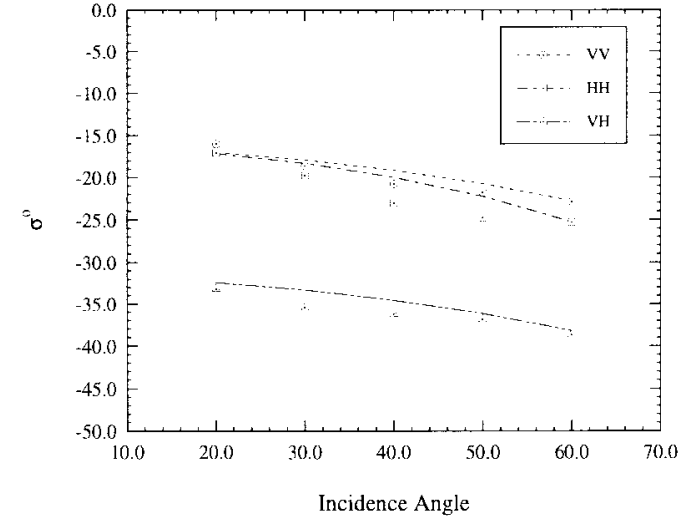

(a)

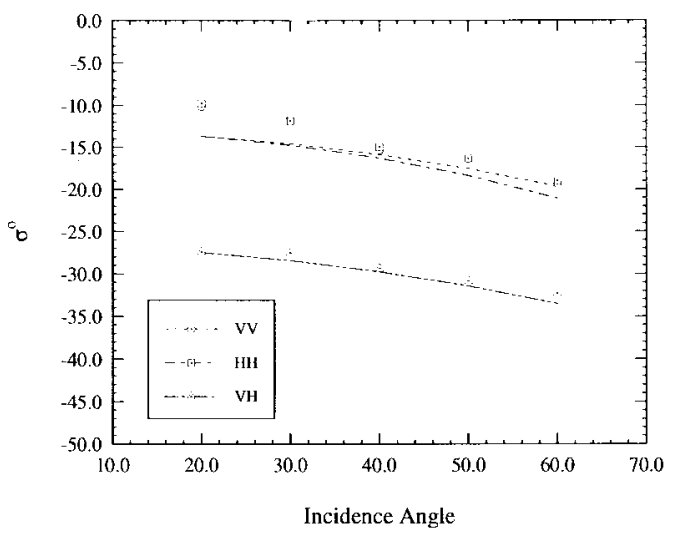

(b)

Fig. 3. (a) Measured (discrete marks) at C-band and (b) predicted (lines) backscatter from bare ground at X-band.

present for all target realizations. That the surface was not isotropic is not a desirable characteristic, but as we will show, the surface, having such a small dielectric constant, does not make an especially large contribution to the backscatter anyway. What is most important is that it cannot be invoked to explain differences in the responses of the various target configurations.

The second type of experiment performed was of a partial diurnal cycle. This experiment took place on March 6, five days after the last experiment in the dry snowpack sequence. In this intervening period, the daily high temperatures had been consistently above freezing, getting as high as $9{ }^{\circ} \mathrm{C}$. As a result, the snowpack had experienced considerable metamorphosis. In addition, there had been deposited about $15 \mathrm{~cm}$ of new snow. Thus, the snowpack had begun to resemble a natural one, complex and difficult to characterize, and mainly unsuitable for comparison with rigorous modeling approaches. However, since copious data on wetness, as a function of both vertical position and time were recorded, along with the polarimetric backscatter at $40^{\circ}$ incidence, it provides an excellent opportunity for direct evaluation of a recent algorithm [7], [8] for inversion of snow wetness levels from target Mueller matrices. A second data set that was recorded on March 1, 1993, in Cadillac, MI, also at $40^{\circ}$ incidence and with complete wetness data, will also be considered.

\section{DRY SNOW BACKSCATTER RESULTS AND DiscusSiON}

\section{A. Bare Ground}

To model the backscatter from the dry (artificial) snow, it is first necessary to account for the contributions of the soil surface below the snowpack as well as the top surface of the snowpack. In [21], it is shown, as the result of an extensive experimental effort, that classical models, such as the small perturbation model and the physical optics and geometric optics solutions of the Kirchhoff approximation, do not accurately predict the copolarized and cross-polarized backscatter from bare soil surfaces. The result of that study is a simple semiempirical model (and inversion algorithm) for copolarized and cross-polarized backscattering coefficients, which uses two parameters: $k s$, the surface roughness, and $m_{v}$, the soil moisture content.

The value of $m_{v}$ for the soil is found to be 0.085 , based on our measured soil dielectric at C-band in conjunction with empirical expressions relating these two quantities, which are provided in [25]. Using this value, along with the soil rms height $s$ (Table I), we may compute predictions of the soil backscatter using the semiempirical model from [21]. Using these exact values, the X-band response is somewhat underestimated; however, a small adjustment of the rms height from the measured value of $0.32-0.37 \mathrm{~cm}$ gives close simultaneous agreement for both frequencies for both copolarized and cross-polarized responses, as shown in Fig. 3. These values, $m_{v}=0.085$ and $s=0.37 \mathrm{~cm}$ will be used to compute the backscatter for the snow-covered ground. The semiempirical surface scattering model from [21] will also be used to compute the backscatter contribution from the top surface of the snow. In this case, the raw measurements were first filtered to remove points that would correspond to Bragg scattering from the periodic disturbance on the surface. For the geometry of this surface, these would occur at incidence angles of 27.8 and $60.4^{\circ}$ for $\mathrm{C}$ - and $\mathrm{X}$-band, respectively, at look angles perpendicular to the direction of the furrows. Beyond this, it is assumed that the backscatter surface response, averaged as it is over a wide range of look directions (on the order of $60^{\circ}$ ) should resemble a randomly rough surface having the same dielectric constant and rms height.

\section{B. Dry Snow Backscatter}

The results from the angular measurements made on three different depths of artificial snow are shown in Fig. 4. Also shown, to better illustrate the snow volume contribution, are simulations of the surface backscatter from 1) the snowcovered ground alone and 2) the snow-covered ground plus the contribution from the top snow surface. These are shown separately to illustrate the relative importance of the topsurface term.

One obvious characteristic of the data is the relatively small dynamic range of the copolarized responses corresponding to different depths. Indeed the copolarized response for the shallowest $(20 \mathrm{~cm})$ depth can be attributed to the effects of the two rough surfaces alone. Still, there is an upward trend apparent, as the 60- and 102-cm depths are on the order 


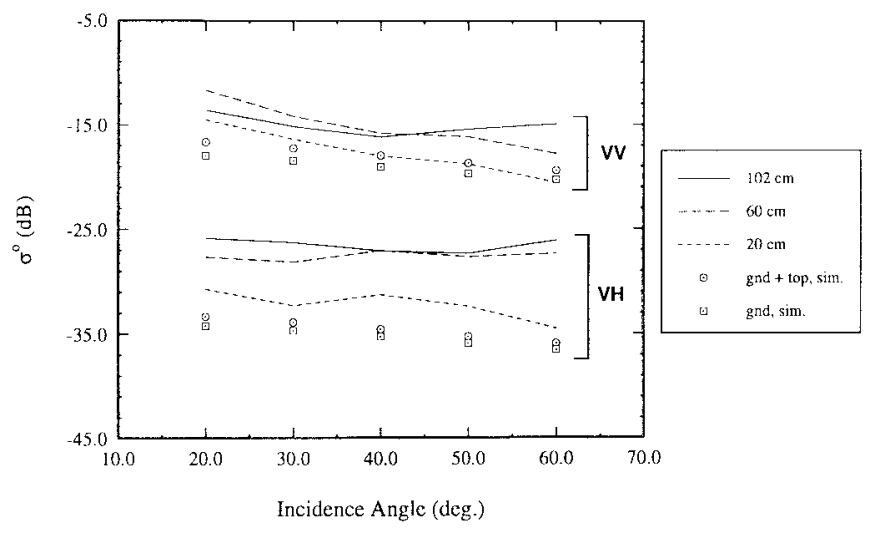

(a)

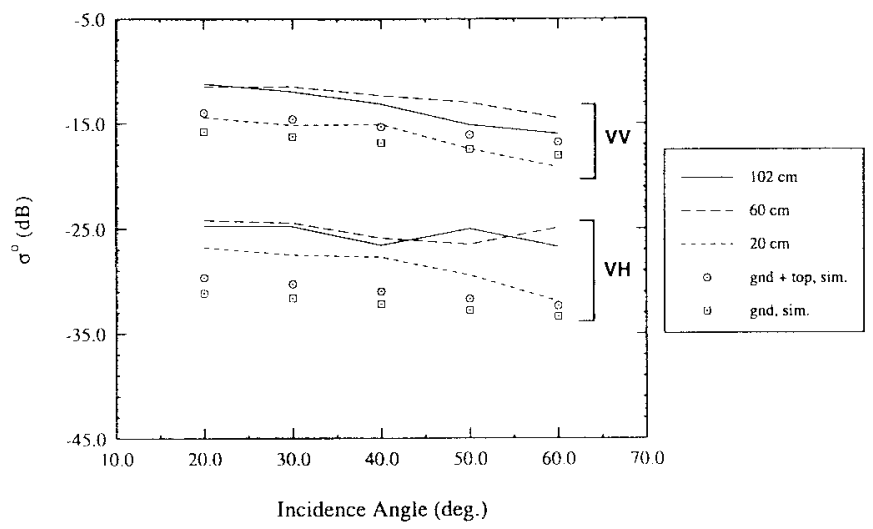

(b)

Fig. 4. Backscatter measurements (VV and $\mathrm{VH}$ ) made of three different snow (artificial) depths at (a) C-band and (b) X-band. Also shown for reference are simulations of the pure ground scattering expected and the contributions from the ground and the snow top surface.

of $3-\mathrm{dB}$ higher than the $20-\mathrm{cm}$ case. The backscatter curves associated with the $60-$ and $102-\mathrm{cm}$ depths criss-cross each other, which is attributed to spatial nonuniformities of the physical characteristics of the target. The fact that the levels are comparable for the two depths implies that $\sigma^{\circ}$ approaches a saturation level corresponding to a half-space condition as the depth exceeds $60 \mathrm{~cm}$.

It is worth comparing these results qualitatively with what has been experimentally observed before for snow in this frequency range. One issue that has been debated in the literature is that of whether snow is observable at all at X-band. Several investigators have claimed that it is not, based on their measurement campaigns [12], [13], whereas, in another case [11], evidence to the contrary is reported. A critical element that must be taken into account in considering this question is the magnitude of the ground scattering. For example, in [12] and [13], the reported rms roughness of the bare ground was $2 \mathrm{~cm}$. Accordingly, the average bare ground scattering level is as high or higher than the maximum levels measured in our present experiments. In the dry snow-pile experiments of [11], the bare ground characteristics are not reported, but $\sigma^{\circ}$ levels for the shallowest snow depths (from Fig. 4 in [11]) are suggestive of a relatively smooth surface, probably similar to that of the present study.
The dynamic range of the cross-polarized response is seen to be considerably greater than that of the copolarized and is unquestionably increased with snow depth, exhibiting an increase on the order of $7 \mathrm{~dB}$. Here too is observed an apparent saturation, as in the case of the copolarized response for the two deepest layers.

Comparison with Discrete Particle-Based Theories: The well-defined nature of the artificial snowpack allows direct comparison with theories that are based on discrete particles. Since intuition suggests that, for particles of this size $(d=0.27$ $\mathrm{mm}$ ) the scattering will be small, we will start by considering an independent scatterer formulation since it is simpler. Since it has been found both experimentally [26] and theoretically [27] that correlated dense medium scattering is less than independent scattering at low frequencies, the solution will represent an upper bound on what DMRT solution techniques may predict.

For a comparison with theory, the complex dielectric constant of the ice particles $\tilde{\varepsilon}_{\text {ice }}$ must also be known. For the real part $\varepsilon_{\text {ice }}^{\prime}$, we use 3.15 . The imaginary part is computed using the following formula [28], which compares very favorably with published data and also accounts for temperature dependance:

$$
\varepsilon_{\text {ice }}^{\prime \prime}=57.34\left(\frac{1}{f}+2.48 \times 10^{-14} \sqrt{f}\right) \exp \left(3.62 \times 10^{-2} T\right)
$$

where $f$ is in $\mathrm{Hz}$ and $T$ is in $\mathrm{K}$. For particles of this size, the scattering albedo $\omega_{0}$, as computed using the Mie solution, is only $5.2 \times 10^{-3}$ for $\mathrm{C}$-band and $2.3 \times 10^{-2}$ for $\mathrm{X}$-band. The scattering albedo is the ratio of the scattering cross section to the total extinction cross section $\left(\kappa_{s} / \kappa_{e}\right)$. That it is so small in this case indicates that volume scattering may be treated as a perturbation on the reduced (by extinction) coherent wave in the medium. Thus, a first-order radiative transfer solution is appropriate for the solution of a layer of these particles. If this solution is computed for a layer of particles (having a smooth surface) over a smooth dielectric half-space, having the same dielectric constant as the soil in the present study, an estimate of the contribution of the snow volume is obtained. When we performed this calculation, as a function of depth and angle for the two frequencies, we found that the maximum copolarized $\sigma^{\circ}$ produced was $-48 \mathrm{~dB}$ at C-band and $-32 \mathrm{~dB}$ at $\mathrm{X}$-band. The contribution to the total scattering represented by these levels does not appear as a visible increase relative to the curve in Fig. 4, which corresponds to the backscattering contributions of the two rough surfaces, top and bottom, alone. The cross-polarized response corresponding to the first-order solution for spherical particles is identical to zero. Use of a more sophisticated radiative transfer solution [18] that uses the discrete ordinate method and so accounts for all orders of scattering gives, not surprisingly, essentially identical results for the copolarized cases; the cross-pol estimate is on the order of $-80 \mathrm{~dB}$ for $\mathrm{C}$-band and $-70 \mathrm{~dB}$ for X-band.

It is obvious that the behavior of the target cannot be explained in terms of the particles of which the snowpack was observed to be comprised. There has appeared in the literature recently work that considers "sticky" particles [29], 


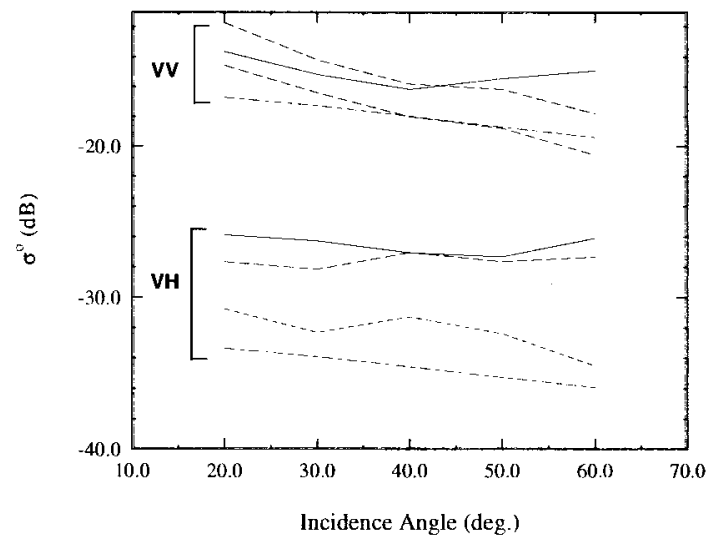

(a)

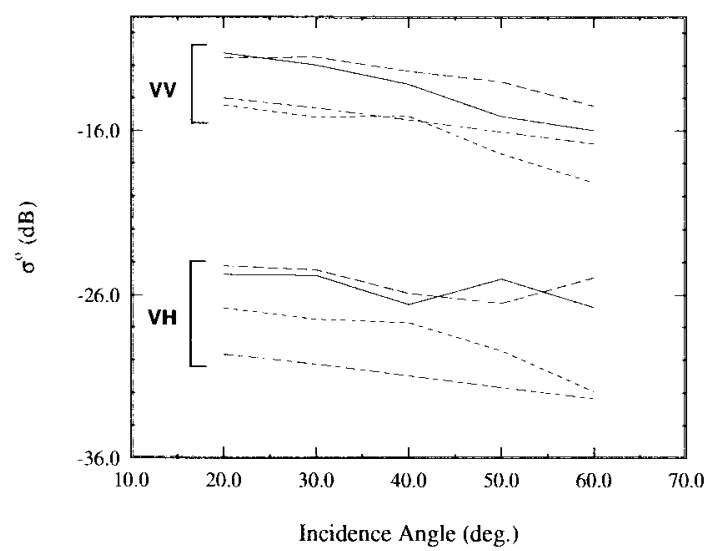

(c)

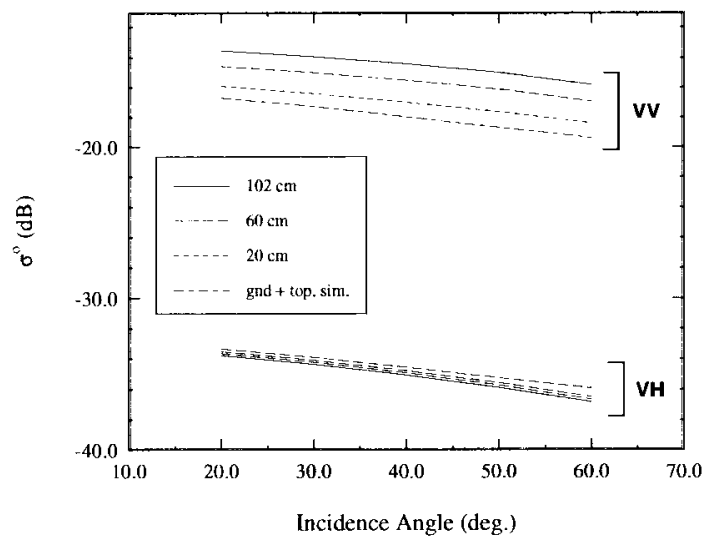

(b)

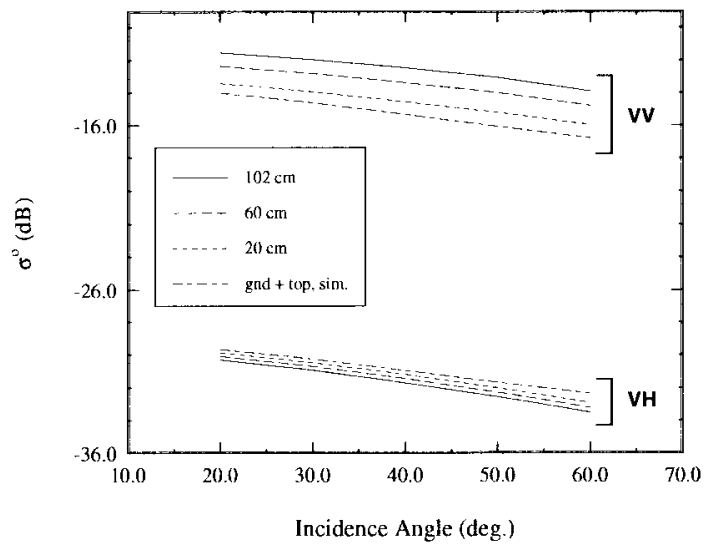

(d)

Fig. 5. Comparison of measured backscatter results for dry (artificial) snow at [(a) and (b)] C-band and [(c) and (d)] X-band with optimal RT predictions obtained by treating the particle size as a free parameter. Optimal snow particle diameters are 1) C-band: $1.7 \mathrm{~mm}$ and 2) X-band: $0.9 \mathrm{~mm}$. Measured snow particle diameter is $0.27 \pm 0.11 \mathrm{~mm}$.

that is, particles that come together to form an aggregate particle, effectively much larger than the individual particles. Obviously, this could have a profound effect upon the scattering behavior of a medium, recalling that in the Rayleigh regime, scattering increases (on a per unit volume basis) as $r^{3}$. If the particle size is treated as a free parameter in the conventional RT formulation, a value can be obtained that gives the optimal agreement with the measured results. The results of such a process are shown in Fig. 5. As shown, reasonable agreement-for the copolarized responses — can be obtained if an effective particle diameter of $1.7 \mathrm{~mm}$ (more than six times larger than the measured average diameter of $0.27 \mathrm{~mm}$ ) is used for C-band and a diameter of $0.9 \mathrm{~mm}$ (more than three times larger than the measured value) is used at $\mathrm{X}$-band. Even in this case, however, the radiative transfer (RT) formulation fails completely to predict the substantial cross-polarized response observed in the measurements.

Faced with the failure of existing particle-based theories to explain the experimental observations, we turn to the question of what useful information can be extracted from this data. A record of the snowpack target that was measured adds, in itself, very little to the study and practice of remote sensing of snow. There is a low probability that the same combination of physical features, including the characteristics on top of the snowpack and beneath it, will be duplicated elsewhere.

A potentially very useful result may be obtained, however, if the intrinsic quantities that specify the extinction and scattering characteristics of this snow material can be retrieved. This concept is illustrated in the following section.

Direct Characterization of the Snow Medium: This section describes an approach for retrieving extinction and scattering parameters for the snow used in this study. A major assumption that is made is that dense media scattering behavior can be described by a first-order radiative transfer formulation. The familiar four terms that result from such a formulation are depicted in Fig. 6. We have intentionally represented the scattering elements as clusters to underscore the point that we are considering "effective" particles in this treatment, which may comprise correlated groups of individual physical particles and/or multiple scattering effects. The validity of such an assumption, that is, that dense media scattering can be understood in terms of a first-order radiative transfer model, has been demonstrated in [30].

A second assumption that will be made, which will greatly simplify the analysis, is that only the direct backscatter term [term (B) in Fig. 6] in the volume scattering formulation is 


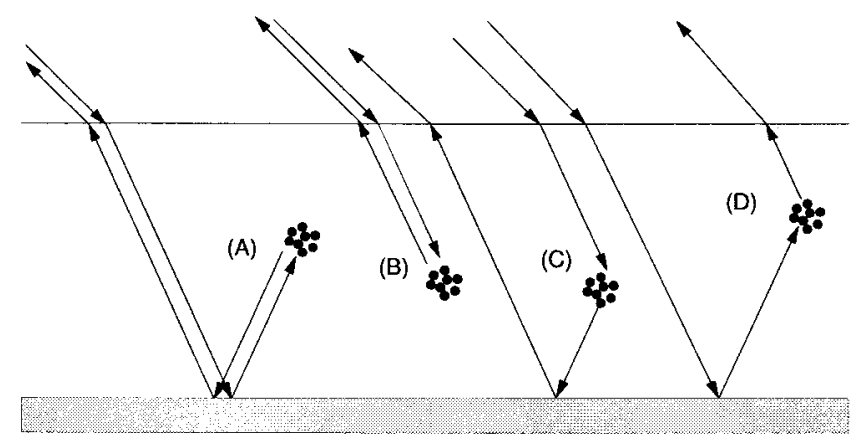

Fig. 6. First-order volume scattering mechanisms in a layer of scatterers.

important. This assumption is justified due to the relatively small reflectivity associated with a reflection at the snow/soil interface, which is less than $-12 \mathrm{~dB}$ for either polarization at any of the angles examined. Since, at least in the case of Rayleigh spheres, the bistatic scattering is always smaller than the backscattering, it follows that the bistatic terms themselves are a minimum of $-12 \mathrm{~dB}$ smaller than the direct term. A complete quantitative analysis of this subject is found in [30, pp. 72-74].

In this case, the first-order solution of the radiative transfer equation reduces to the form

$$
\mathcal{L}_{m}=\mathcal{L}_{\mathrm{ts}}+\frac{1}{\mu_{\mathrm{o}}^{\prime}} \mathcal{T}_{21}\left[\gamma \mathcal{P}_{\mathrm{bs}}+\frac{\mathcal{L}_{\mathrm{gnd}}}{L^{2}}\right] \mathcal{T}_{12}
$$

where $\mathcal{L}_{\text {ts }}$ is the Mueller matrix corresponding to the top surface scattering, $\mathcal{T}_{p q}$ is the surface intensity transmissivity matrix [31, p. 145] for transmission from $p$ to $q$, where one corresponds to the air and two to the snow medium, $\mu_{\mathrm{o}}^{\prime}$ is the cosine of the refracted angle, $\mathcal{P}_{\mathrm{bs}}$ is the unknown backscatter component of the phase matrix for the effective volume scattering element, $\mathcal{L}_{\text {gnd }}$ corresponds to the rough surface below, and

$$
\begin{aligned}
\gamma & =\frac{1-\exp \left(-2 \kappa_{e} d / \mu_{\mathrm{o}}^{\prime}\right)}{2 \kappa_{e} / \mu_{\circ}^{\prime}} \\
L^{2} & =\exp \left(2 \kappa_{e} d / \mu_{\circ}^{\prime}\right)
\end{aligned}
$$

where $\kappa_{e}$ is the extinction and $d$ is the layer depth. This model has in the past been applied in a scalar sense to both snow [11] and vegetation [32]. In order to apply (2) in a vector sense, it is necessary to construct Mueller matrices corresponding to the top and bottom rough surfaces. In the preceding analysis, we had used a semiempirical model for rough surface scattering because of its greater reliability. The model as it has been presented is a scalar one; in [33], however, Oh extends this approach to include an empirical model for $\alpha$, the degree of correlation between $S_{\mathrm{vv}}$ and $S_{\mathrm{hl}}$. This parameter governs [34] the width of the probability density function (pdf) that describes the distribution $\phi_{\mathbf{h h}}-\phi_{\mathrm{vv}}$, the phase difference of the copolarized complex scattering amplitudes. Oh's formula is given as

$$
\alpha=\frac{1}{2}\left[1-0.2(\sin \theta)^{A\left(k s, \Gamma_{\circ}\right)}\right](\cos \theta)^{B\left(k s, \Gamma_{\circ}\right)}
$$

where

$$
\begin{aligned}
& A\left(k s, \Gamma_{\circ}\right)=\left(16.5 \Gamma_{\circ}+5.6\right) \exp \left[-41.6 k s \Gamma_{\circ}^{2}\right] \\
& B\left(k s, \Gamma_{\circ}\right)=8.1 \Gamma_{\circ} k s \exp [-1.8 k s]
\end{aligned}
$$

where $\Gamma_{0}$ is the Fresnel reflectivity at nadir and $k s$ is as before, the roughness of the surface, which is expressed as the product of the wavenumber in the background medium and the rms height of the surface. The importance of knowing $\alpha$ for the present purpose is that it is expressible in terms of the elements of the Mueller matrix and, thus, can aid us in constructing these matrices for the ground and the snow surface. The quantity $\alpha$ is formally given by [34] as

$$
\alpha=\frac{1}{2}\left[\frac{\left(M_{33}+M_{44}\right)^{2}+\left(M_{34}-M_{43}\right)^{2}}{M_{11} M_{22}}\right]^{1 / 2}
$$

where $M_{m n}$ are elements of the modified Mueller matrix $\mathcal{L}_{m}$, as shown in (7) at the bottom of the page.

From (6), if we make the assumption that $M_{43}$, which corresponds (for backscatter) to the term $\left\langle\operatorname{Im}\left(S_{\mathrm{vv}} S_{\mathbf{h h}}^{*}\right)\right\rangle$, is much smaller than $\left\langle\operatorname{Re}\left(S_{\mathrm{vV}} S_{\mathrm{hh}}^{*}\right)\right\rangle$, then

$$
\left\langle\operatorname{Re}\left(S_{\mathrm{vV}} S_{\mathrm{hh}}^{*}\right)\right\rangle \approx \pm \alpha \sqrt{M_{11} M_{22}} \text {. }
$$

From comparison with the bare ground backscatter data, the selection of the negative root is indicated (following the forward scatter alignment (FSA) convention [31]), which is also in agreement with polarimetric predictions for rough surfaces [31] in which it has been found that the statistical phase difference between $S_{\mathrm{Vv}}$ and $S_{\mathrm{hh}}$ is near $180^{\circ}\left[0^{\circ}\right.$ in the backscatter alignment (BSA) convention]. For the bare ground under the snow layer, $\alpha$ was found using (3) to be essentially unity for both C-band and X-band for all angles between 20 and $60^{\circ}$. For the top snow surface, $\alpha$ varied from unity at $20^{\circ}$ to 0.89 at $60^{\circ}$ for both frequencies. A further assumption that was made in constructing the Mueller matrices for the top and bottom surfaces - and one which is reasonable given the above discussion regarding the copolarized phase difference for rough surfaces-is that the elements $M_{34}$ and $M_{43}$ corresponding to $\pm \operatorname{Im}\left\langle S_{\mathrm{vv}} S_{\mathbf{h h}}^{*}\right\rangle$ are zero.

The polarimetric character of the two rough interfaces, above and below the snow layer, are thus characterized by a target Mueller matrix having the (FSA) form shown in (9) at the bottom of the next page, where

$$
g_{\mathrm{pq}}=\frac{\sigma_{\mathrm{pq}}^{\circ}}{4 \pi \cos \theta_{\mathrm{im}}}
$$

$$
\mathcal{L}_{m}=\left[\begin{array}{cccc}
\left\langle\left|S_{\mathrm{vv}}\right|^{2}\right\rangle & \left\langle\left|S_{\mathrm{hv}}\right|^{2}\right\rangle & \left\langle\operatorname{Re}\left(S_{\mathrm{vh}}^{*} S_{\mathrm{vv}}\right)\right\rangle & \left\langle-\operatorname{Im}\left(S_{\mathrm{vh}}^{*} S_{\mathrm{vv}}\right)\right\rangle \\
\left\langle\left. S_{\mathrm{hv}}\right|^{2}\right\rangle & \left\langle\left|S_{\mathrm{hh}}\right|^{2}\right\rangle & \left\langle\operatorname{Re}\left(S_{\mathrm{hh}}^{*} S_{\mathrm{hv}}\right\rangle\right. & \left\langle-\operatorname{Im}\left(S_{\mathrm{hv}}^{*} S_{\mathrm{hh}}\right)\right\rangle \\
\left\langle 2 \operatorname{Re}\left(S_{\mathrm{vv}} S_{\mathrm{hv}}^{*}\right)\right\rangle & \left\langle 2 \operatorname{Re}\left(S_{\mathrm{vh}} S_{\mathrm{hh}}^{*}\right)\right\rangle & \left\langle\operatorname{Re}\left(S_{\mathrm{vv}} S_{\mathrm{hh}}^{*}+S_{\mathrm{vh}} S_{\mathrm{hv}}^{*}\right)\right\rangle & \left\langle-\operatorname{Im}\left(S_{\mathrm{vv}} S_{\mathrm{hh}}^{*}-S_{\mathrm{vh}} S_{\mathrm{hv}}^{*}\right)\right\rangle \\
\left\langle 2 \operatorname{Im}\left(S_{\mathrm{vv}} S_{\mathrm{lv}}^{*}\right)\right\rangle & \left\langle 2 \operatorname{Im}\left(S_{\mathrm{vh}} S_{\mathrm{hh}}^{*}\right)\right\rangle & \left\langle\operatorname{Im}\left(S_{\mathrm{vv}} S_{\mathrm{hh}}^{*}+S_{\mathrm{vh}} S_{\mathrm{hv}}^{*}\right)\right\rangle & \left\langle\operatorname{Re}\left(S_{\mathrm{vv}} S_{\mathrm{hh}}^{*}-S_{\mathrm{vh}} S_{\mathrm{hv}}^{*}\right)\right\rangle
\end{array}\right]
$$




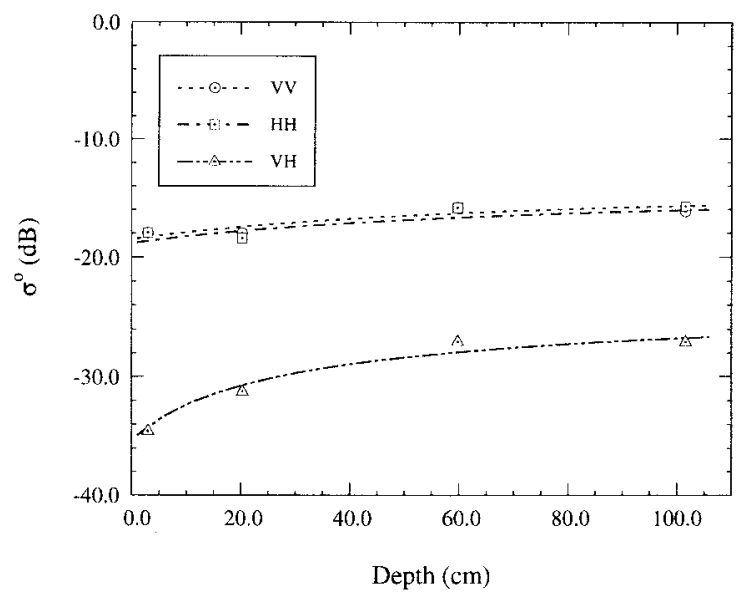

(a)

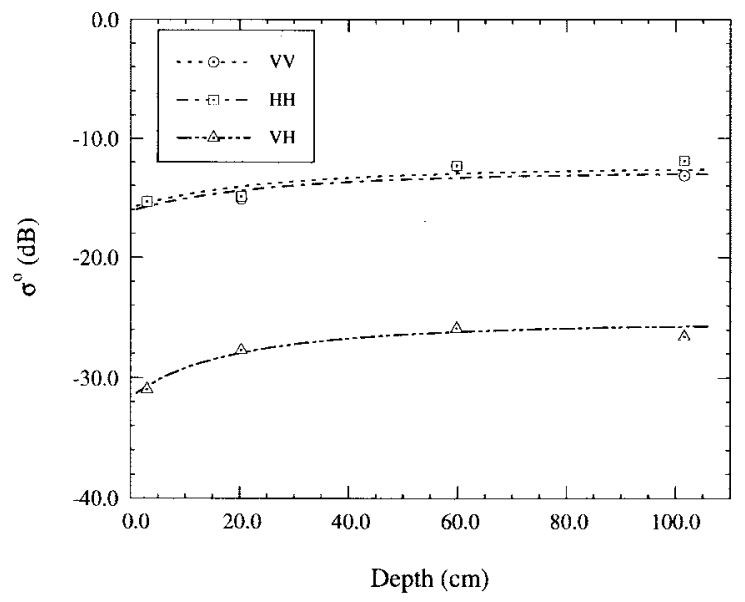

(b)

Fig. 7. Comparison of snow data to radiative transfer-type model computations for (a) C-band and (b) X-band. Parameters for model are obtained through an optimization process that uses this data.

with $\sigma_{\mathrm{pq}}^{\circ}$, as given by the semiempirical model of [21] and $\theta_{\mathrm{im}}$, the angle of incidence onto the surface in the appropriate medium (air for the top surface, snow for the underlying soil surface). The correlation coefficient $\alpha$ is as calculated in (3).

The parameters to be specified through an optimization of the measured polarimetric data with the model given in (2) are the scalar extinction and the backscatter component of the phase matrix $\mathcal{P}_{\mathrm{bs}}$. Since the snow is assumed to be an isotropic medium, we use the form

$$
\mathcal{P}_{\mathrm{bs}}=\left[\begin{array}{cccc}
P 1 & P 2 & 0 & 0 \\
P 2 & P 1 & 0 & 0 \\
0 & 0 & P 3-P 2 & -P 4 \\
0 & 0 & P 4 & P 3+P 2
\end{array}\right]
$$

which requires the volume backscattering for the two copolarized channels to be identical. The nature of the other elements is set by considerations of reciprocity $\left(\left|S_{\mathrm{vh}}\right|^{2}=\left|S_{\mathrm{hv}}\right|^{2}\right)$ and zero correlation between the copolarized and cross-polarized complex scattering amplitudes. With the unknown scalar extinction, there are therefore five unknown quantities to be determined to characterize the snow polarimetrically. The estimation of these parameters is done using an optimization package suitable for nonlinear problems [35]. The optimization is performed using only the $40^{\circ}$ data, considering all three depths for both $\mathrm{C}$-band and $\mathrm{X}$-band and then the results applied to generate predictions for the other cases (depths and angles) not used in the optimization process.

In Figs. 7-9, we show the measured data along with predictions generated using the estimated parameters $\kappa_{e}$ and $P_{1}, \ldots, P_{4}$ in (2). The angular variation predicted by (2) (in which the angular variation is contained in the transmissivity matrices) appears reasonable, as does the behavior, with respect to depth at angles that were not involved in the optimization process. The estimated values for the parameters $\kappa_{e}$ and $P_{1}, \ldots, P_{4}$ for both $\mathrm{C}$ - and $\mathrm{X}$-band are summarized in Table II. A comparison of the values for extinction obtained by this empirical and two theoretical methods is given in Table III. One of the two methods, the effective field approximation (EFA), is associated with conventional radiative transfer [36] (CRT) and the other, quasicrystalline approximation-coherent potential (QCA_CP) [37], is used in DMRT. As seen in Table III, and for the scattering computations mentioned earlier, the predicted effects of the snowpack are practically negligible.

Since the analysis was a complete polarimetric one, we can examine the results, with respect to the two remaining (given the two copolarized responses and the cross-polarized response already examined) independent quantities associated with a measured Mueller matrix in the backscatter direction. These quantities are the degree of correlation $\alpha$, which was introduced earlier, and another quantity that pertains to the position of the maximum of the pdf [34] describing the distribution of the phase difference between $S_{\mathrm{vv}}$ and $S_{\mathrm{hh}}^{*}$. This quantity is known as the copolarized phase difference and is denoted by the symbol $\zeta$.

Fig. 10 compares the measured values of these quantities at C-band and the corresponding estimates generated through the use of the RT model, with parameters given in Table II for the case of the $60-\mathrm{cm}$ artificial snow layer. The agreement is not exceptionally good. This is attributed to 1) the need to specify the polarimetric character of both rough surface (above and below the snow) and 2) the generally noisy character of the data.

\section{Discussion of Dry Snow Results}

The most important result from the dry snow experiments was the observation that the most widely used models de-

$$
\mathcal{L}_{\text {surf }}=\left[\begin{array}{cccc}
g_{\mathrm{vv}} & g_{\mathrm{vh}} & 0 & 0 \\
g_{\mathrm{vh}} & g_{\mathrm{hh}} & 0 & 0 \\
0 & 0 & -\alpha \sqrt{g_{\mathrm{vv}} g_{\mathrm{hh}}}-g_{\mathrm{vh}} & 0 \\
0 & 0 & 0 & -\alpha \sqrt{g_{\mathrm{vv}} g_{\mathrm{hh}}}+g_{\mathrm{vh}}
\end{array}\right]
$$




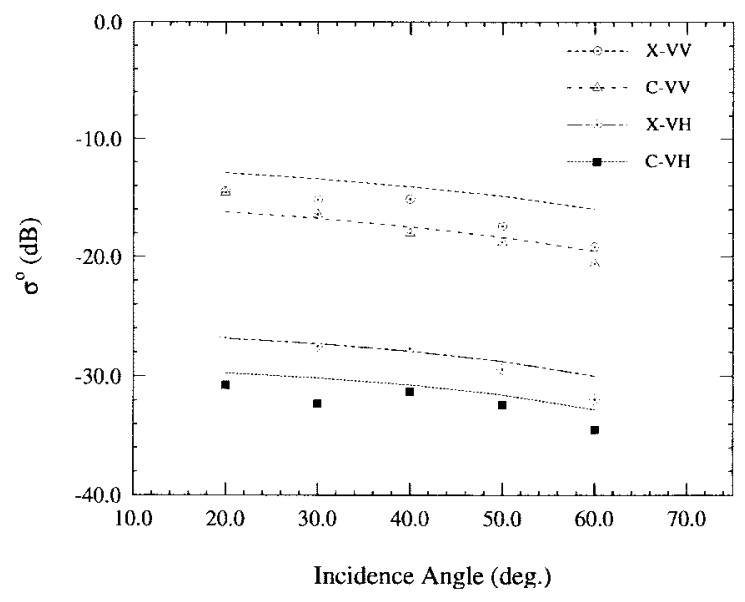

(a)

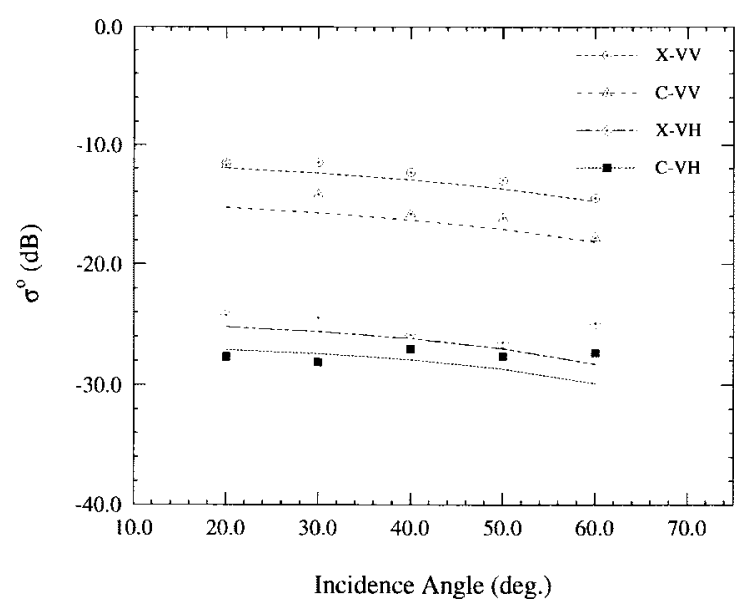

(b)

Fig. 8. Comparison of $\mathrm{C}$-band and $\mathrm{X}$-band snow data to radiative transfer-type model predictions for a (a) $20-\mathrm{cm}$ snow layer and (b) $60-\mathrm{cm}$ snow layer. Model is parameterized through analysis of $40^{\circ}$ data.

scribing the electromagnetic scattering characteristics of dense media failed to give reasonable predictions in comparison to measured data. While one experiment is not sufficient for evaluating the validity of a theory, the present experiment carries significant weight because of the relatively simple and well-characterized nature of the target snowpack.

In the subsequent analysis, a simple method for retrieving electromagnetic parameters, intrinsic to the snow medium itself was described. It is noteworthy-in the face of the very large body of research that has been done on snow and pertaining directly to snow-that this marks the first time an attempt has been made to characterize the effect of a snow medium considered in isolation from other effects in a polarimetric way. The only other comparable example is the snow-pile experiments and subsequent scalar analysis described in [11], in which the radar response of a snow pile of varying depths was collected. That effort was hampered by a number of departures from ideal experimental circumstances. For example, only one spatial sample was available, the snow was artificially heaped up by mechanical means, and efforts to characterize the upper or lower surfaces or even the general uniformity of the snow pile were absent.

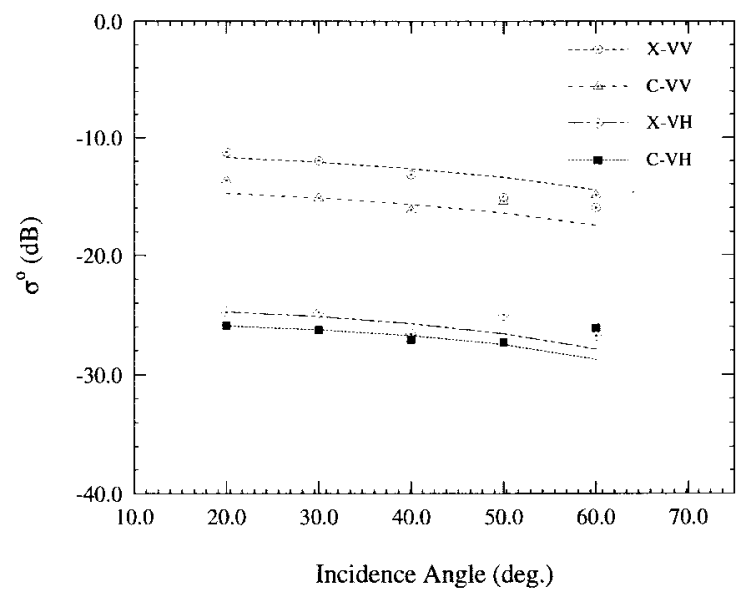

Fig. 9. Comparison of $\mathrm{C}$-band and $\mathrm{X}$-band snow data to radiative transfer-type model predictions for a 102-cm layer. Model is parameterized through analysis of $40^{\circ}$ data.

TABLE II

Artificial SNOW: EMPIRICAL PARAMETERS FOR RT-MODEL

\begin{tabular}{c|c|c}
\hline \hline Parameter & C-band & X-band \\
\hline$\kappa_{e}(\mathrm{~Np} / \mathrm{m})$ & 0.513 & 1.28 \\
\hline $\mathbf{P}_{1}$ & $0.316 \times 10^{-2}$ & $0.128 \times 10^{-1}$ \\
\hline $\mathbf{P}_{2}$ & $0.330 \times 10^{-3}$ & $0.740 \times 10^{-3}$ \\
\hline $\mathbf{P}_{3}$ & $-0.169 \times 10^{-3}$ & $-0.976 \times 10^{-3}$ \\
\hline $\mathbf{P}_{4}$ & $0.531 \times 10^{-3}$ & $0.220 \times 10^{-2}$ \\
\hline
\end{tabular}

TABLE III

Estimates of the EXtinction (IN NP/M) OF the Artificial SNOW

\begin{tabular}{c|l|l}
\hline \hline Source & C-band & X-band \\
\hline "Meas." & 0.513 & 1.28 \\
\hline EFA & 0.020 & 0.047 \\
\hline QCA-CP & 0.042 & 0.096 \\
\hline
\end{tabular}

Of course, for direct characterization of snow, it is not feasible to use the approach employed in this experiment. A practical scheme should borrow from the spirit of the snowpile experiments alluded to above, in terms of having a target of a fairly manageable size-except that a turntable could be employed to allow realization of independent samples and steps taken to control the character of the surfaces above and below. The material representing the underlying half-space could be chosen to best facilitate the retrieval of information. Feasibility studies on this technique, using not snow but stable materials like sand and gravel, have in fact been carried out by the author, and the results, including an analysis of the validity of a vector radiative transfer model with empirically derived parameters for describing very dense media, may be found in [30].

\section{DIURNAL RESULTS AND DISCUSSION}

This section presents the results from measurements of partial diurnal cycles that were collected on two separate occasions, as described in the Section II. The Snow Probe was used on both occasions to record vertical profiles of the liquid water content as a function of time. The two snowpacks examined had very different physical descriptions, as will be shown. This allows some insight into the generation and spatial 


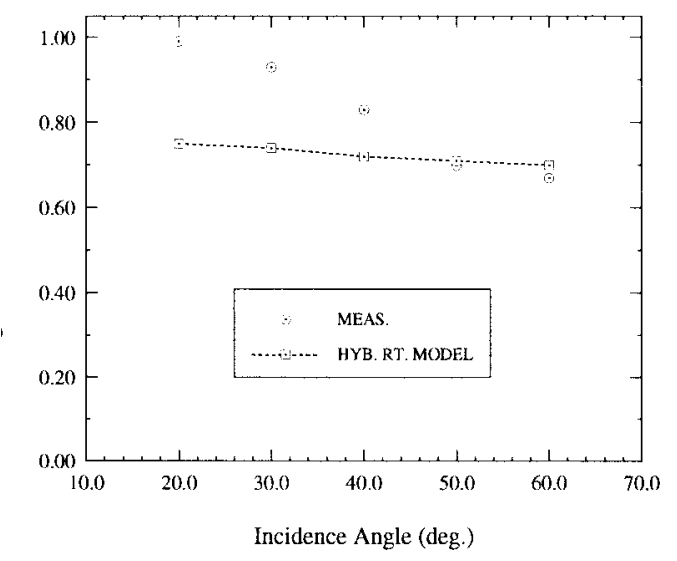

(a)

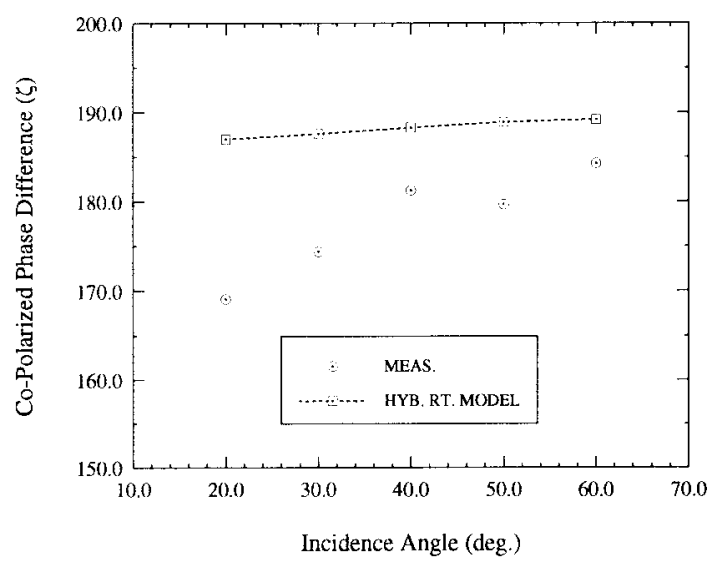

(b)

Fig. 10. Phase statistics for the $60-\mathrm{cm}$ artificial snow layer. Shown are the measured (C-band) values and those calculated using an RT model with associated parameters given in Table II.

behavior of liquid water in a snowpack. We will use the diurnal results and associated wetness data to evaluate a particular algorithm that has been recently proposed for the retrieval of snow liquid water content.

\section{A. Brighton Diurnal Results}

In the Brighton diurnal experiment, the measurements were made continuously from 10 a.m. until 7 p.m. Overnight temperatures prior to the experiment were well below freezing; the temperatures during the day ranged from $-6^{\circ} \mathrm{C}$ at 8 a.m. to more than $6{ }^{\circ} \mathrm{C}$ at 3 p.m. It was a very sunny day, and heavy melting was evident. By 6 p.m., the temperature dropped abruptly below freezing again. The target snowpack was 0.88 $\mathrm{m}$ deep, with about $15 \mathrm{~cm}$ of relatively fresh snow on top, however, the bottom $16 \mathrm{~cm}$ consisted of solid ice ${ }^{1}$. The density of the snowpack was about $0.25 \mathrm{~g} / \mathrm{cm}^{3}$ at the top, increasing linearly to 0.45 by $30 \mathrm{~cm}$ into the pack, where it remained essentially constant to the bottom of the snowpack.

The copolarized and cross-polarized results for $\mathrm{C}$ - and $\mathrm{X}$ band are shown in Fig. 11. Both frequencies show a significant reduction in the backscatter at midday, very typical of a

\footnotetext{
${ }^{1}$ At the onset of warmer weather, the radar truck had been moved from the spot of the dry snow experiments to a location that happened to be near the base of one of the ski runs. The drainage from this slope was the cause of the large ice accumulation at the base of the snowpack.
}

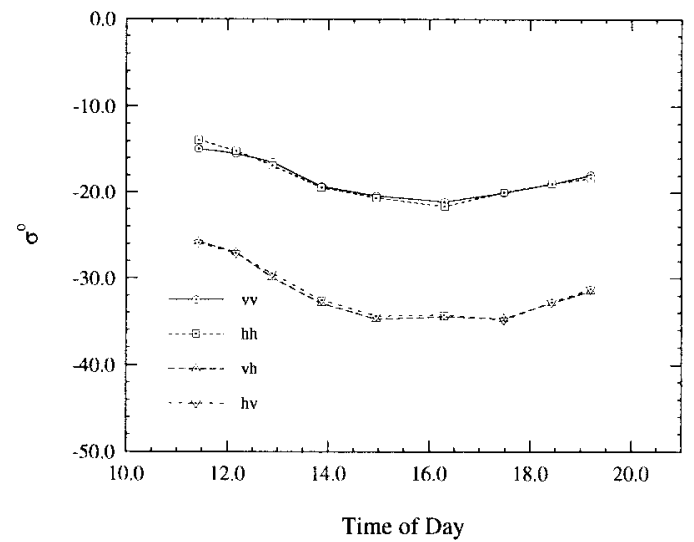

(a)

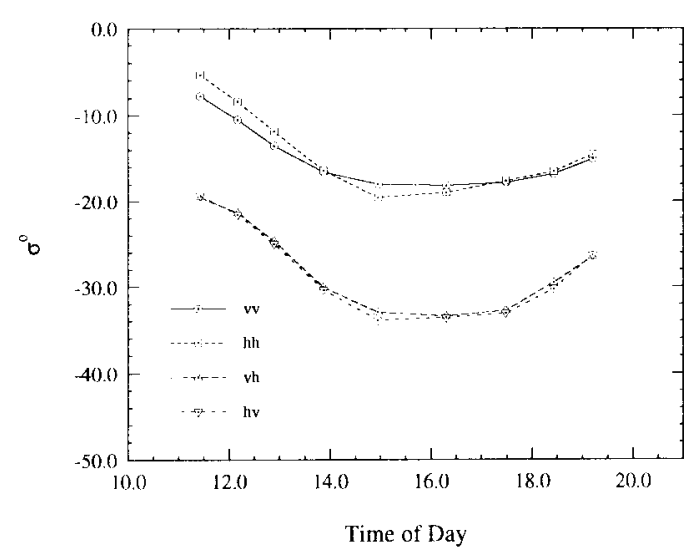

(b)

Fig. 11. Copolarized and cross-polarized backscatter results for (a) C-band and (b) X-band from Brighton (partial) diurnal experiment. Incidence angle is $40^{\circ}$.

diurnal response, in this case $8-10 \mathrm{~dB}$ for $\mathrm{C}$-band and $\approx 14$ $\mathrm{dB}$ for $\mathrm{X}$-band. By the end of the measurements, however, the radar response at both frequencies appears to be headed back up toward the original morning levels. The results of the snow probe measurements made concurrently with the radar measurements are shown in Fig. 12. The wetness measurements begin at $18 \mathrm{~cm}$, due to the presence of the ice layer below this, and are made at roughly $5-\mathrm{cm}$ intervals. The temporal spacing between measurements of the vertical profile was $\approx 1 \mathrm{~h}$. What is most striking about the wetness map is the presence of very significant wetness levels in the lower 35-40 $\mathrm{cm}$ of the snowpack, even at the earliest point measured in the morning, while the top surface is completely dry. The wetness level of the top surface remains fairly moderate throughout the day, staying below 5\% except, curiously, at the very end of the day, just before the temperature fell very swiftly below freezing again. It would appear that the top of the snowpack was freely draining throughout the day as the wetness levels toward the bottom were observed to increase to maximal snow wetness levels $>12 \%$.

\section{B. Cadillac Diurnal Results}

In the Cadillac diurnal experiment, the snowpack was only $22-\mathrm{cm}$ deep. The measurements were made from 10 a.m. 


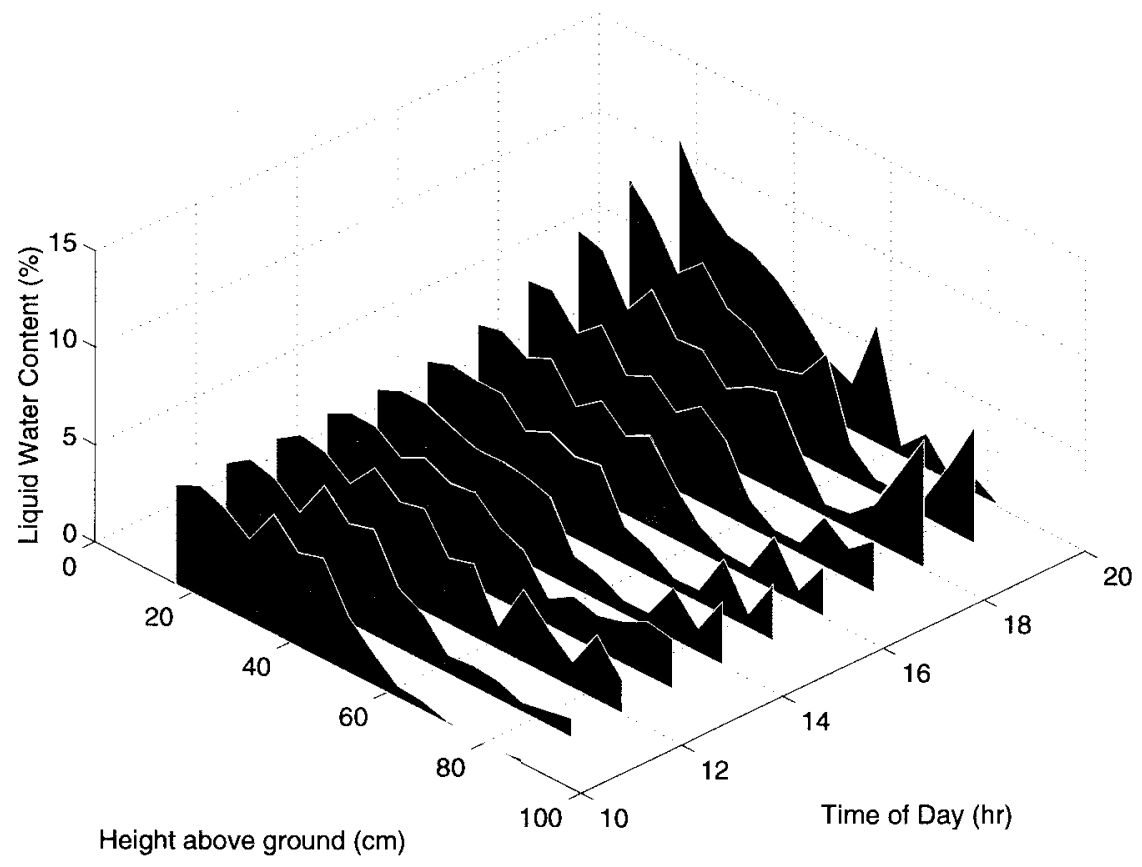

Fig. 12. Vertical profiles of snow wetness measured as a function of time. Measurements were taken with the snow probe during the Brighton diurnal experiment.

to about 4 p.m. As in the Brighton case, the overnight temperatures had been subfreezing, but the experiment day itself became very warm, reaching $12{ }^{\circ} \mathrm{C}$ for a high and very sunny. Extensive melting was clearly evident around the entire experimental area.

The copolarized and cross-polarized results are shown in Fig. 13 and the associated wetness map in Fig. 14. The apparent higher frequency of collection of radar measurements relative to the Brighton data pertains to the number of measurements that were combined as a single data point in each case. These results are seen to have a very different character than that observed in the Brighton case. An important feature of the Cadillac snowpack was the presence, at least during the initial few hours of the experiment, of a very prominent ice lens, starting approximately $2.5 \mathrm{~cm}$ below the top surface and having a thickness of about $2 \mathrm{~cm}$. A result that is attributable to both this feature and the relatively much warmer temperatures that occurred compared to the Brighton case is the presence of very high wetness levels in the uppermost levels of the snowpack. Though the high wetness levels are not strictly confined to the $2.5 \mathrm{~cm}$ above the ice lens, it is apparent that the ice lens, particularly early on and to a lesser extent as it became softer and more permeable, impeded the drainage of the liquid water through the snowpack. Indeed there appears to be some evidence from an examination of Fig. 14 that the ice lens rapidly evolved as it softened into a region supporting very high wetness levels.

One implication of this situation (high wetness levels near the surface) evident in the backscatter (Fig. 13) is a "hump," or at least a temporary departure from the downward trend of $\sigma^{\circ}$, for both copolarized and cross-polarized channels. This feature, occurring between 11:30 a.m. and about 2 p.m., is

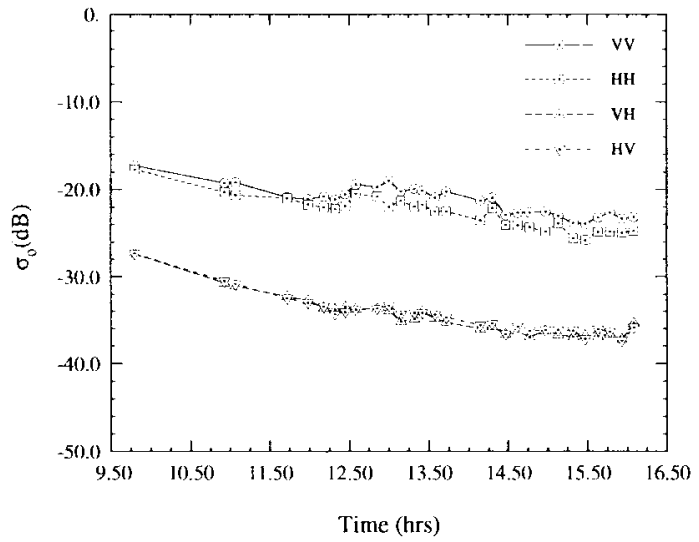

(a)

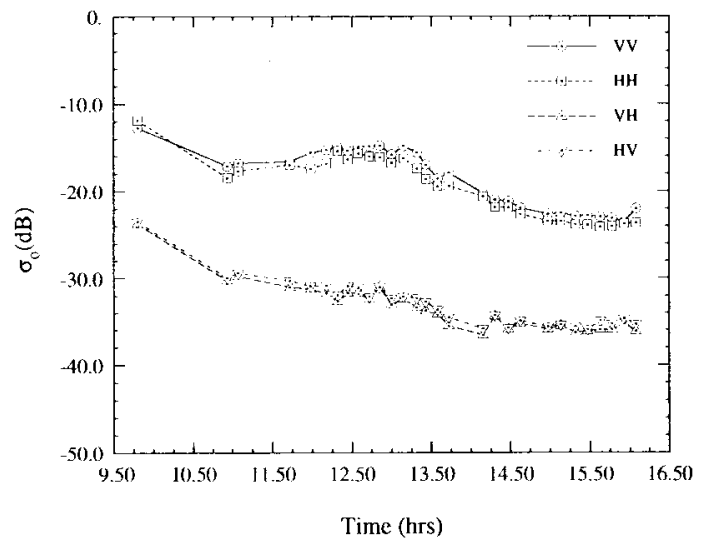

(b)

Fig. 13. Copolarized and cross-polarized backscatter results for a (a) C-band and (b) X-band from Cadillac (partial) diurnal experiment. Incidence angle is $40^{\circ}$. 


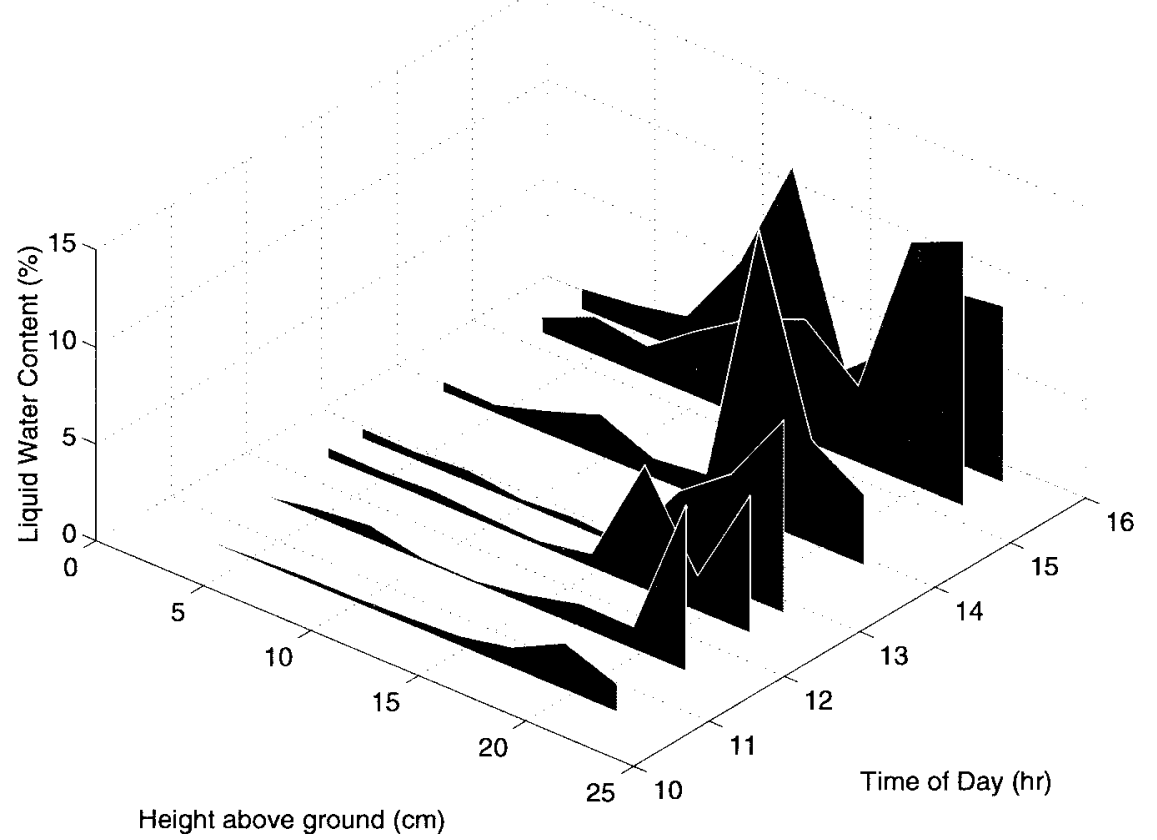

Fig. 14. Vertical profiles of snow wetness measured as a function of time. Measurements were taken with the snow probe during the Cadillac diurnal experiment.

apparently tied to the increase in surface scattering, due to a higher dielectric contrast, balancing the reduction in volume scattering.

\section{Evaluation of Wetness Retrieval Algorithm}

The availability of detailed snow wetness information along with polarimetric backscatter data allows comparison with an algorithm that has recently been developed [7], [8], for the retrieval of snow liquid water estimates from polarimetric data at C-band. The ability to detect, quantitatively, from remotely sensed data, a parameter, such as liquid-water content in snow, would constitute a very important achievement and a major step forward in supplying hydrologists and other earth scientists with data products critical for various applications. It is all the more impressive given the difficulties associated with even direct measurements of liquid water content. Given the potential value of such an algorithm, it is highly desirable that its validity be supported by careful ground-based measurement efforts. The present diurnal experiment appears to be an excellent candidate for this evaluation.

The algorithm was originally motivated by an AIRSAR data set that was collected over glaciers in the Öztal Alps, Austria. Prior to these measurements, physical characteristics, such as snow depth, density, wetness, and surface roughness, were measured. The final version of the algorithm, as appears in [8], was developed in conjunction with data from a recent (April 1994) SIR-C/X-SAR mission taken over Mammoth Mountain on the eastern slope of the Sierra Nevadas. A similar ground truth campaign was employed in this effort as well.

The algorithm conceives of the radar response as an incoherent addition of volume and surface responses. No attempt is made to explicitly model the magnitude of the volume contribution to the scattering. Instead, it is shown how, un- der certain assumptions, the relationships between certain quantities in the polarimetric volume radar response may be explicitly constrained. Specifically, the volume, since wet snow is assumed, is considered a half-space. A first-order volume scattering mechanism is assumed, which leads to the result that the ratio of the copolarized volume responses is equal to the square of the ratio of the Fresnel transmissivities corresponding to each polarization, respectively. Similar ratios are constructed between the copolarized volume responses and a term associated with the correlation between the two copolarized channels

$$
\sigma_{\mathrm{vvhh}}^{v}=\left\langle\operatorname{Re}\left[S_{\mathrm{vv}}^{v} S_{\mathrm{hh}}^{v *}\right]\right\rangle
$$

where $S_{\mathrm{vV}}$ and $S_{\mathrm{hh}}$ are the $\mathrm{VV}$ and $\mathrm{HH}$ complex scattering amplitudes, respectively, associated with the volume. Their correlation is assumed to be unity. The only unknown parameter in these ratios formed is the permittivity of the snow.

The surface radar response is directly modeled using an empirical expression that is based on predictions of the integral equation method (IEM) surface scattering model [38] computed over the range of surface parameters expected for snow. A correlation term is formed as for the volume case; once again, the correlation factor is considered to be unity. The unknowns in the empirical surface scattering models are the permittivity of the snow and a general surface roughness term.

Two equations are formed involving the surface scattering responses and the ratios of the volume responses. These equations are combined into a single equation (22) in [8], which is only a function of the snow permittivity $\varepsilon_{s}$. The inversion algorithm amounts to finding the value of $\varepsilon_{s}$ that most nearly satisfies this equation. 


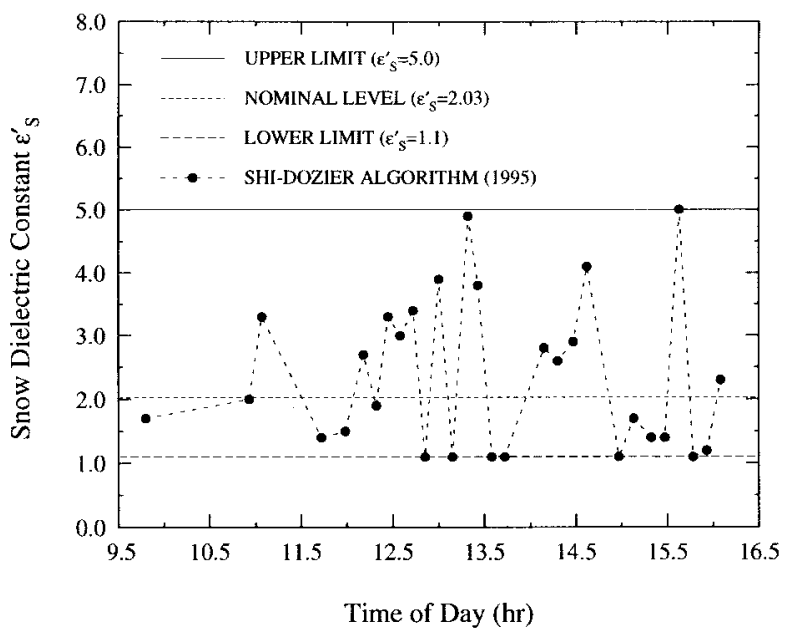

Fig. 15. Application of inversion algorithm for snow wetness applied to Cadillac diurnal data set. Shown is the actual output from the algorithm, snow permittivity $\varepsilon_{s}$. The "nominal level" shown is based on the expected dry snow permittivity that is based on ground truth measurements of density.

Application to Measured Diurnal Data: We first attempted to treat the Cadillac data set with this algorithm. The physical description of the snowpack in the Cadillac experiment seemed to agree generally with the inherent assumptions of the model, namely, $m_{v}$ effects manifesting themselves primarily in the upper regions of the snowpack. Only the C-band data were used in the algorithm evaluation, which consists of testing a range of values of $\varepsilon_{s}$ to find the value most nearly satisfying (22) in [8]. We used a range that corresponded to values of this parameter that might reasonably be associated with snow $1.1 \leq \varepsilon_{s} \leq 5.0$.

The values of $\varepsilon_{s}$ that were the outputs of the algorithm, with respect to the Cadillac data, are shown in Fig. 15. As seen, most of the 29 separate sets of polarimetric radar measurements resulted in estimates of $\varepsilon_{s}$, which are within the bounds of "reasonable" results that we set. Only one data set produced a value pegged at the top of the range $\left(\varepsilon_{s}=5.0\right)$, and six were pegged at the bottom $\left(\varepsilon_{s}=1.1\right)$. Also shown in the figure is the nominal value of the permittivity of the top layer of the snowpack, based on ground truth measurements. The average measured density of the top layer was found to be about $0.5 \mathrm{~g} \mathrm{~cm}^{-3}$; such a density would correspond to a $d r y$ snow dielectric constant of 2.03 , which is the nominal value shown in the figure.

As described in [19], the permittivity of wet snow is modeled as a dry snow value given by

$$
\varepsilon_{\mathrm{ds}}^{\prime}=1+1.7 \rho_{\mathrm{ds}}+0.7 \rho_{\mathrm{ds}}^{2}
$$

which depends on density $\rho_{\mathrm{ds}}$ alone plus an incremental increase, which depends on snow wetness $\left(m_{v}\right)$ alone. This incremental increase in the permittivity is given by [39]

$$
\Delta \varepsilon_{s}^{\prime}=0.02 m_{v}^{1.015}+\frac{0.073 m_{v}^{1.31}}{1+\left(f_{s} / f_{w}\right)^{2}}
$$

where $f_{s}$ is the frequency at which the permittivity is measured, $f_{w}=9.07 \mathrm{GHz}$ is the relaxation frequency of water at $0{ }^{\circ} \mathrm{C}$, and $m_{v}$ is expressed in percent.

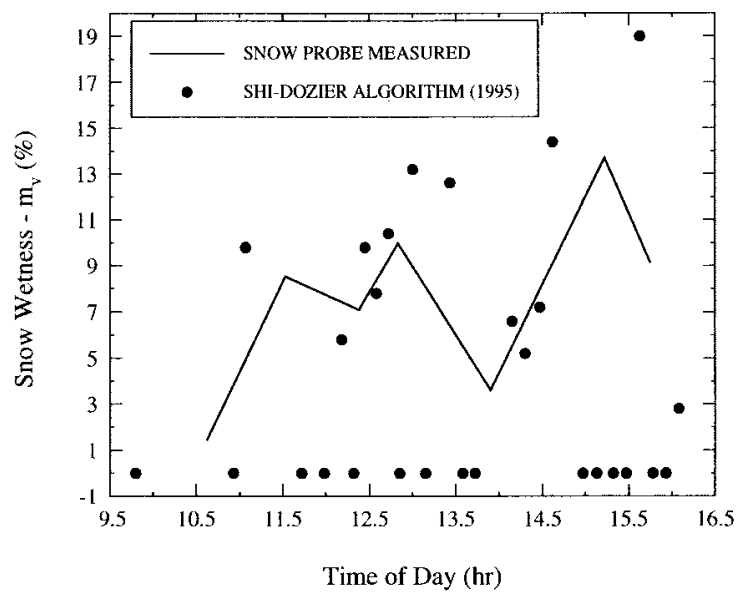

Fig. 16. Snow wetness inversion algorithm results from Cadillac diurnal data set compared with actual measured values of $m_{v}$ in the uppermost layer of the snowpack.

If the incremental increase is known, an accurate estimate of $m_{v}$ may be obtained by inverting (14). The incremental increase itself may really only be known if the density of the snow is also known, so that the base contribution to $\varepsilon_{s}$ represented by the dry snow component $\varepsilon_{\mathrm{ds}}$ may be accounted for. Then the incremental increase is given by

$$
\Delta \varepsilon_{s}^{\prime}\left(m_{v}\right)=\varepsilon_{s}^{\prime}\left(m_{v}, \rho\right)-\varepsilon_{\mathrm{ds}}^{\prime}(\rho) .
$$

This is one shortcoming of the algorithm by [8], and one that is not addressed by the authors, namely, the relatively large errors that result in trying to estimate $m_{v}$ of snow from a measurement of $\varepsilon_{s}$ alone, with no knowledge of the snow density. The density component in the calculation of the dielectric constant of wet snow accounts for about $43 \%$ of the total variation that may occur in this property (wet snow dielectric constant). A detailed discussion of these errors is found in [30, p. 105].

For the purpose of evaluating the present algorithm, however, we will compute estimates of $m_{v}$ for the Cadillac data set by subtracting the value of 2.03 (based on the measured density of $0.5 \mathrm{~g} \mathrm{~cm}^{-3}$ ) from each of the algorithmestimated permittivities shown in Fig. 15. The remainder of this operation is the incremental increase in the permittivity due to water, and this quantity can be inverted to obtain an estimate of $m_{v}$. These estimates are shown in Fig. 16 along with the values of $m_{v}$, which were measured in the uppermost layer as a function of time using the Snow Probe. All cases in which the algorithm estimated value of $\varepsilon_{s}$ are less than the nominal dry-snow value of 2.03 are considered as having $m_{v}=0$. It is seen that the algorithm gives a reasonable performance in terms of its predictions that there were very high snow wetness levels present. About half of the cases examined result in estimates of $m_{v}=$ 0 . Of the cases that give nonzero estimates of $m_{v}$, very high wetness levels are indicated-similar to but in general exceeding the actual measurements-which may be seem to-very roughly - follow the trends, as a function of time, which were observed in the measurements. 


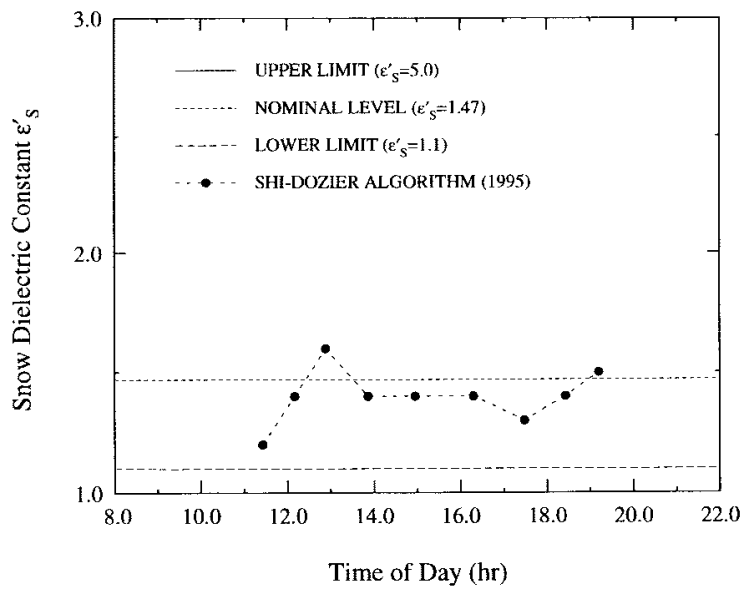

Fig. 17. Application of inversion algorithm for snow wetness applied to Brighton diurnal data set. Shown is the actual output from the algorithm, snow permittivity $\varepsilon_{s}$. The "nominal level" shown is based on the expected dry snow permittivity that is based on ground truth measurements of density.

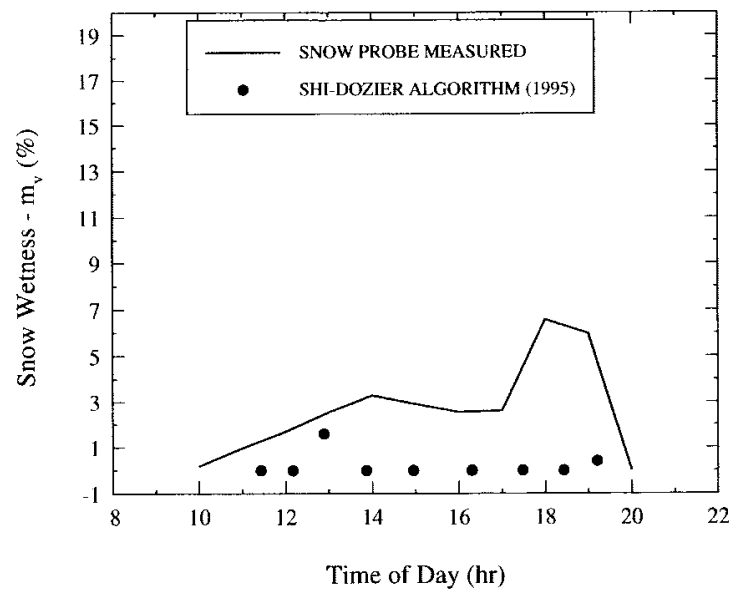

Fig. 18. Snow wetness inversion algorithm results from Brighton diurnal data set compared with actual measured values of $m_{v}$ in the uppermost layer of the snowpack.

As an additional test, we applied the algorithm to the Brighton diurnal data set, for which, as was seen in Fig. 18, the top layer of the snowpack had relatively low levels of liquid water content. The values of $\varepsilon_{s}$, which we obtained from the application of the inversion algorithm to this data set, are shown in Fig. 17. The "nominal value" of $\varepsilon_{s}$ indicated in this figure is based on the measured density of about $0.25 \mathrm{~g}$ $\mathrm{cm}^{-3}$ in the uppermost layer of the snowpack. As can be seen, most of the estimated values are lower than this nominal level, but not by much. In particular, none of the nine separate cases treated resulted in permittivity estimates outside of the range $\left(1.1 \leq \varepsilon_{s} \leq 5.0\right)$, which are "reasonable". Also, the relatively very low estimates relative to those shown for the Cadillac case in Fig. 15 demonstrate the algorithm is genuinely sensitive to this parameter.

The associated estimates of $m_{v}$, generated in the same manner as was described for the Cadillac case above, that is, using our knowledge of the actual snow density in the topmost layer of the snowpack, are shown in Fig. 18. Only two of the nine cases examined give nonzero estimates of the snow liquid water content. The actual measured values, however, are relatively low, with the possible exception of the values measured at about 6 p.m. (18:00 h), which are seen to be above $5 \%$ liquid water content. This condition itself was a rather anomalous one, occurring as it did just prior, due the refreezing of the snowpack top surface.

General Comments on the Wetness Retrieval Algorithm: The central concept behind this algorithm appears to be that the relationships among the quantities $\sigma_{\mathrm{vv}}, \sigma_{\mathrm{hh}}$, and $\sigma_{\mathrm{vvhh}}$ (the latter of which is related to the correlation between the copolarized responses) for volume scattering can be distinguished from the corresponding relationships of these quantities associated with surface scattering. That this should be the case is not obvious. With respect to the copolarized responses, for both surface and volume scattering, it is generally the case that the vv-polarized response exceeds the hh-response increasingly more as incidence angle increases. With respect to the quantity $\sigma_{\mathrm{vvhh}}$, it has previously been noted by both [7] (the authors of the inversion algorithm) and others $[4]^{2}$ that the correlation coefficient for rough surface scattering is approximately unity. In addition, in the wetness inversion algorithm, the authors implicitly use a correlation coefficient of unity for the volume scattering as well. Thus, it is difficult to see how the polarization relationships between the polarimetric quantities in volume and surface scattering, respectively, could allow discrimination between these two scattering mechanisms.

Despite these questions about the fundamental concepts upon which the algorithm is constructed, it must be said that its performance, with respect to the two quite different data sets of this present study - the Cadillac and Brighton diurnal experiments-is fairly impressive. Using only polarimetric radar data, the algorithm gave estimates of dielectric constant that were fairly comparable to those directly measured. In addition, when provided with density values for the two respective snowpacks, the algorithm was able to produce estimates of liquid water content reasonably close to those found to be present by direct measurements.

On the Question of the Behavior of $\sigma^{\circ}$ with Increasing $m_{v}$ : The authors of this wetness inversion algorithm make a case for the increase in the backscatter levels for wet snow. While it is true that such a phenomenon is readily predicted theoretically, it has only been observed experimentally in some exceptional cases. The Cadillac scenario might be considered such a case, where a combination of circumstances (ice lens near the surface and exceptionally warm weather) produced, to a small degree, a surface scattering effect. In general, however, the depression of the radar backscatter level has been almost universally observed in practice [10], [12], [13], [15], [24] at both microwave (including the present study) and millimeter-wave frequencies. In [12], examples are given of the opposite case, of increasing $\sigma^{\circ}$, but these cases were brought on by the presence of rain in a time frame very close to the measurements in one case, the presence of hail in another case, and in another case, wet snow that was roughened with a shovel. The rain scenario, in fact, mirrors

\footnotetext{
${ }^{2}$ Specifically addresses the coefficient of variation for rough surfaces, which as we have indicated, carries essentially identical information as the correlation coefficient.
} 
the circumstances under which the experiment in the Öztal Alps was conducted-an experiment that produced the data in which the inversion algorithm was originally based.

The preponderance of evidence, with respect to a decreased backscatter, suggests that models of wet snow may not be realistic. Possible explanations for the departure from theoretical predictions may be that 1) liquid water drains from the upper levels of the snowpack before it becomes abundant enough to increase the dielectric constant significantly or, as may well be the case with the Cadillac data, 2) drainage from the immediate top of the layer helps to create a sort of matching layer, leading to small surface scattering even for high wetness levels near the top surface.

\section{SUMMARY}

This paper has described the results of two types of polarimetric radar experiments that were carried out on snowpacks.

In Section III, we presented results and analysis for measurements that were made at $\mathrm{C}$ - and $\mathrm{X}$-band on the bare ground and then three successively deeper $(20,60$, and $102 \mathrm{~cm})$ layers of artificial dry snow. The details of the physical character of the snowpack and the environmental conditions associated with the experiments made these results especially amenable to comparison with discrete particle-based theoretical modeling techniques. It was shown, however, that these techniques did not provide reasonable agreement with the experimental observations. A subsequent analysis of the data was presented by which polarimetric scattering and extinction quantities intrinsic to the snow medium were retrieved.

In Section IV, results for backscatter collected during partial diurnal cycles were presented along with the results of extensive measurements of snow liquid water content, which were made concurrently. These data were used in an attempt to confirm the validity of an algorithm that has recently been developed by [8] for the retrieval of snow liquid water content from polarimetric C-band measurements. Although an examination of the conceptual framework of the algorithm reveals certain basic assumptions that seem difficult to justify, its performance, with respect to the two separate diurnal data sets, is fairly impressive. The algorithm was able to correctly characterize the Cadillac and Brighton snowpacks (top layer) as very wet and reasonably dry, respectively.

\section{REFERENCES}

[1] J. Koskinen, L. Kurvonen, V. Jäskeläinen, and M. Hallikainen, "Capability of radar and microwave radiometer to classify snow types in forested areas," in Proc. IGARSS'94, vol. II, p. 1283.

[2] F. T. Ulaby, P. Siqueira, and K. Sarabandi, "A hybrid electromagneticstatistical approach for characterizing MMW scattering by terrain," in AGARD Conf. Proc. 501, 1993.

[3] R. M. Narayanan and S. R. Jackson, "Snow cover classification using millimeter-wave radar imagery," in Proc. IGARSS'94, vol. IV.

[4] J. J. van Zyl, H. A. Zebker, and C. Elachi, "Imaging radar polarization signatures: Theory and observation," Radio Sci., vol. 22, no. 4, pp. 529-543, 1987.

[5] J. J. van Zyl, "Unsupervised classification of scattering behavior using radar polarimetry data," IEEE Trans. Geosci. Remote Sensing, vol. 27, pp. 36-45, Jan. 1989.

[6] D. L. Evans, T. G. Farr, J. J. van Zyl, and H. A. Zebker, "Radar polarimetry: Analysis tools and applications," IEEE Trans. Geosci. Remote Sensing, vol. 26, pp. 774-789, Nov. 1988.
[7] J. Shi and J. Dozier, "Radar backscattering response to wet snow," in Proc. IGARSS'92, vol. II, p. 927.

[8] _ "Inferring snow wetness using C-band data from SIR-C's polarimetric synthetic aperture radar," IEEE Trans. Geosci. Remote Sensing, vol. 33, pp. 905-914, July 1995.

[9] D. T. Davis, Z. Chen, L. Tsang, J. N. Hwang, and A. T. C. Chang, "Retrieval of snow parameters by iterative inversion of neural network," IEEE Trans. Geosci. Remote Sensing, vol. 31, pp. 842-852, July 1993.

[10] W. H. Stiles and F. T. Ulaby, "The active and passive microwave response to snow parameters 1. Wetness," J. Geophys. Res., vol. 85, no. C2, pp. 1037-1044, 1980.

[11] F. T. Ulaby and W. H. Stiles, "The active and passive microwave response to snow parameters 2 . Water equivalent of dry snow," $J$. Geophys. Res., vol. 85, no. C2, pp. 1045-1049, 1980.

[12] C. Mätzler, E. Schanda, and W. Good, "Toward the definition of optimum sensor specifications for microwave remote sensing of snow," IEEE Trans. Geosci. Remote Sensing, vol. GE-20, pp. 57-66, Jan. 1982.

[13] C. Mätzler and E. Schanda, "Snow mapping with active microwave sensors," Int. J. Remote Sensing, vol. 5, no. 2, pp. 409-422, 1984.

[14] A. T. C. Chang, J. L. Foster, M. Owe, and D. K. Hall, "Passive and active microwave studies of wet snowpack properties," Nordic Hydrol., vol. 16, pp. 57-66, 1985.

[15] F. T. Ulaby, T. F. Haddock, R. T. Austin, and Y. Kuga, "Millimeterwave radar scattering from snow: 2. Comparison of theory with experimental observations," Radio Sci., vol. 26, no. 2, pp. 343-351, 1991.

[16] J. B. Mead, P. S. Chang, S. P. Lohmeier, P. M. Langlois, and R. McIntosh, "Polarimetric observations and theory of millimeter-wave backscatter from snow cover," IEEE Trans. Antennas Propagat., vol. 41, pp. 38-46, Jan. 1993.

[17] M. T. Hallikainen, F. T. Ulaby, and T. E. Van Deventer, "Extinction behavior of dry snow in the 18- to 90-GHz range," IEEE Trans. Geosci. Remote Sensing, vol. GE-25, pp. 737-745, Jan. 1987.

[18] Y. Kuga, F. T. Ulaby, T. F. Haddock, and R. D. DeRoo, "Millimeterwave radar scattering from snow: 1 . Radiative transfer model," Radio Sci., vol. 26, no. 2, pp. 329-341, 1991.

[19] J. R. Kendra, F. T. Ulaby, and K. Sarabandi, "Snow probe for in situ determination of wetness and density," IEEE Trans. Geosci. Remote Sensing, vol. 32, pp. 1152-1159, Nov. 1994

[20] M. A. Tassoudji, K. Sarabandi, and F. T. Ulaby, "Design consideration and implementation of the LCX polarimetric scatterometer (polarscat)," Radiation Lab., Univ. Michigan, Ann Arbor, Tech. Rep. Rep. 022486T-2, 1989.

[21] Y. Oh, K. Sarabandi, and F. T. Ulaby, "An empirical model and an inversion technique for radar scattering from bare soil surfaces," IEEE Trans. Geosci. Remote Sensing, vol. 30, pp. 370-381, Mar. 1992.

[22] K. Sarabandi, Y. Oh, and F. T. Ulaby, "Measurement and calibration of differential Mueller matrix of distributed targets," IEEE Trans. Antennas Propagat., vol. 40, pp. 1524-1532, Dec. 1992.

[23] D. R. Brunfeldt, "Theory and design of a field-portable dielectric measurement system," in Proc. IGARSS'87, vol. I, pp. 559-563.

[24] P. Chang, J. Mead, R. McIntosh, R. Davis, and H. Boyne, "A detailed study of the backscatter characteristics of snowcover measured at 35, 95, and 225 GHz," in Proc. IGARSS'94, vol. IV, p. 1932.

[25] M. T. Hallikainen, F. T. Ulaby, M. C. Dobson, M. A. El-Rayes, and L. Wu, "Microwave dielectric behavior of wet soil-Part I: Empirical models and experimental observations," IEEE Trans. Geosci. Remote Sensing, vol. GE-23, pp. 25-34, Jan. 1985.

[26] A. Ishimaru and Y. Kuga, "Attenuation constant of a coherent field in a dense distribution of particles," J. Opt. Soc. Amer., vol. 72, pp. 1317-1320, 1982.

[27] L. Tsang and J. A. Kong, "Scattering of electromagnetic waves from a dense medium consisting of correlated Mie scatterers with size distributions and applications to dry snow," J. Electromagn. Waves Applicat., vol. 6, no. 3, pp. 265-296, 1992.

[28] E. Nyfors, "On the dielectric properties of dry snow in the $800 \mathrm{MHz}$ to 13 GHz range," Radio Lab., Helsinki Univ. Technol., Helsinki, Finland, Tech. Rep. S135, 1982.

[29] L. M. Zurk, K. H. Ding, L. Tsang, and D. P. Winebrenner, "Monte Carlo simulations of the extinction rate of densely packed spheres with clustered and nonclustered geometries," in Proc. IGARSS'94, vol. I, p. 535.

[30] J. R. Kendra, "Microwave remote sensing of snow: An empirical/theoretical scattering model for dense random media," Ph.D. dissertation, Univ. Michigan, Ann Arbor, 1995.

[31] F. T. Ulaby and C. Elachi (Eds.), Radar Polarimetry for Geoscience Applications. Dedham, MA: Artech House, 1990.

[32] E. P. W. Attema and F. T. Ulaby, "Vegetation modeled as a water cloud," Radio Sci., vol. 13, no. 2, pp. 357-364, 1978. 
[33] Y. Oh, K. Sarabandi, and F. T. Ulaby, "An empirical model for phase difference statistics of rough surfaces," in Proc. IGARSS'93.

[34] K. Sarabandi, "Derivation of phase statistics from the Müeller matrix," Radio Sci., vol. 27, no. 5, pp. 553-560, 1992.

[35] J. L. Zhou and A. L. Tits, "User's guide for FSQP version 3.3b: A Fortran code for solving constrained nonlinear (minimax) optimization problems, generating iterates satisfying all inequality and linear constraints," Syst. Res. Center, Univ. Maryland, College Park, Tech. Rep. TR-92-107r3, Sept. 1993.

[36] L. Tsang, J. A. Kong, and R. T. Shin, Theory of Microwave Remote Sensing. New York: Wiley Interscience, 1985 .

[37] B. Wen, L. Tsang, D. P. Winebrenner, and A. Ishimaru, "Dense medium radiative transfer theory: Comparison with experiment and application to microwave remote sensing and polarimetry," IEEE Trans. Geosci. Remote Sensing, vol. 28, pp. 46-59, Jan. 1990

[38] A. K. Fung, Z. Li, and K. S. Chen, "Backscattering from a randomly rough dielectric surface," IEEE Trans. Geosci. Remote Sensing, vol. 30, pp. 365-396, Mar. 1992.

[39] M. Hallikainen, F. T. Ulaby, and M. Abdelrazik, "Dielectric properties of snow in the 3 to $37 \mathrm{GHz}$ range," IEEE Trans. Antennas Propagat., vol. AP-34, pp. 1329-1339, Nov. 1986.

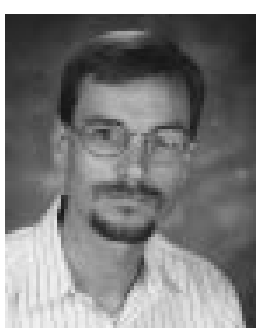

John R. Kendra (S'92-M'95) received the B.S.E.E. degree from the University of Houston, Houston, TX, in 1989. He received the M.S.E.E. and Ph.D. degrees in electrical engineering from The University of Michigan, Ann Arbor, in 1990 and 1995, respectively. His dissertation dealt with experimental and theoretical studies of microwave scattering from dense random media, especially pertaining to snow. One aspect of this research was the development of an electromagnetic sensor, the Snow Probe, for in situ determination of snow

liquid water content

He is currently employed at Raytheon E-Systems, Dallas, TX, where he develops image processing algorithms for remote-sensing applications and algorithms pertaining to the calibration of multispectral and panchromatic sensors.

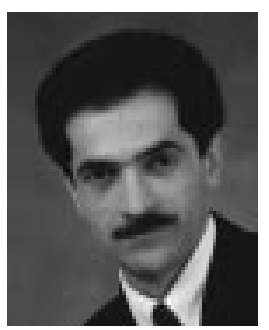

Kamal Sarabandi (S'87-M'90-SM'92) received the B.S. degree in electrical engineering from Sharif University of Technology, Tehran, Iran, in 1980. He received the M.S.E. degree in electrical engineering in 1986, and the M.S. degree in mathematics and the $\mathrm{Ph} . \mathrm{D}$. degree in electrical engineering in 1989, all from The University of Michigan, Ann Arbor.

$\mathrm{He}$ is currently an Associate Professor in the Department of Electrical Engineering and Computer Science, University of Michigan. He has 15 years of experience with microwave sensors and radar systems. In the past seven years, he has served as the Principal Investigator and Co-Investigator on many projects sponsored by NASA, JPL, ARO, ONR, and GM, all related in one way or another to microwave and millimeter-wave radar remote sensing. He has published many book chapters and more than 70 papers in refereed journals on electromagnetic scattering, random media modeling, microwave measurement techniques, radar calibration, application of neural networks in inverse scattering problems, and microwave sensors. $\mathrm{He}$ has also had more than 130 papers and invited presentations in national and international conferences and symposia on similar subjects.

Dr. Sarabandi is listed in Who's Who in Electromagnetics. He served as the Chairman of the Geoscience and Remote Sensing Society Southeastern Michigan chapter from 1991 to 1997. He is also a member of Commission $\mathrm{F}$ of URSI and of the Electromagnetic Academy. He was a recipient of a 1996 Teaching Excellence Award and the 1997 Henry Russel Award from the Regent of The University of Michigan.

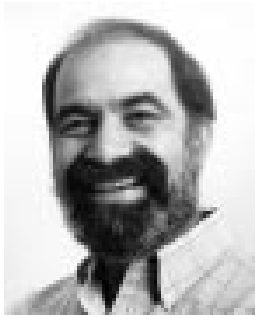

Fawwaz T. Ulaby (M'68-SM'74-F'80) received the B.S degree in physics from the American University, Beirut, Lebanon, in 1964 and the M.S. and $\mathrm{Ph} . \mathrm{D}$. degrees in electrical engineering from the University of Texas, Austin, in 1966, and 1968, respectively.

$\mathrm{He}$ is the Williams Distinguished Professor of Electrical Engineering and Computer Science and Director of the Radiation Laboratory, The University of Michigan, Ann Arbor. His current interests include microwave and millimeter-wave remote sensing, radar systems, and radio wave propagation. He has authored eight books and published over 400 papers and reports on these subjects.

Dr. Ulaby is the recipient of numerous awards, including the IEEE Geoscience and Remote Sensing Distinguished Achievement Award in 1983, the IEEE Centennial Medal in 1984, The American Society of Photogrammetry's Presidential Citation for Meritorious Service in 1984, the NASA Group Achievement Award in 1990, and The University of Michigan Distinguished Faculty Achievement Award in 1991. He served as President of the IEEE Geoscience and Remote Sensing Society from 1980 to 1982, as Executive Editor of the IEEE Transactions on Geoscience and Remote Sensing from 1983 to 1985 , and as General Chairman of several international symposia. He is a member of the National Academy of Engineering and serves on several scientific boards and professional committees. 